\title{
Random Order Congestion Games
}

\author{
Michal Penn \\ Faculty of Industrial Engineering and Management, Technion, Haifa 32000, Israel \\ email: mpenn@ie.technion.ac.il \\ Maria Polukarov \\ School of Electronics and Computer Science at University of Southampton, SO17 1BJ, Southampton, UK \\ email: mp3@ecs.soton.ac.uk \\ Moshe Tennenholtz \\ Microsoft Israel R\&D Center, 13 Shenkar Street, Herzeliya 46725, Israel \\ Faculty of Industrial Engineering and Management, Technion, Haifa 32000, Israel \\ email: moshet@microsoft.com,moshet@ie.technion.ac.il
}

\begin{abstract}
We introduce a new class of games, Random Order Congestion Games [ROCGs]. In an ROCG, each player has a task that can be carried out by any element of a set of resources, and each resource executes its assigned tasks in a random order. Each player's aim is to minimize his expected cost which is the sum of two terms - the sum of the fixed costs over the set of his utilized resources and the expected cost of his task execution. The cost of a player's task execution is determined by the earliest time his task is completed, and thus it might be beneficial for him to assign his task to several resources. We prove the existence of pure strategy Nash equilibria in ROCGs. Moreover, we present a polynomial time algorithm for finding such an equilibrium in a given ROCG.
\end{abstract}

Key words: congestion games; asynchronous distributed systems; random order of task execution; pure-strategy Nash equilibrium; algorithms

MSC2000 Subject Classification: Primary: 91A10, 91A40; Secondary: 91A06, 91A80

OR/MS subject classification: Primary: Games/group decisions - Noncooperative; Secondary: Computers/computer science - Artificial intelligence

1. Introduction. Congestion games received a lot of attention in the recent game theory and computer science literature [4, 5, 8, 9, 10. In a classic congestion game [16, each player chooses a subset of a set of available resources in order to perform his task. The cost of using a particular resource is determined by its congestion. The important property of congestion games is that they possess pure strategy Nash equilibria. Monderer and Shapley [10] introduced the notions of potential function and potential game and proved that the existence of a potential function implies the existence of a pure strategy Nash equilibrium. They also showed that the classes of finite potential games and congestion games coincide.

Classic congestion games can be viewed as synchronous: the cost suffered by a player when selecting a particular resource is determined only by the number of users who have chosen that resource, and does not take into account the actual order in which the assigned tasks are executed. In this paper ${ }^{1}$ we present a new class of games - random order congestion games (ROCGs) - that model noncooperative congestion settings in which resources execute their assigned tasks in a randomly chosen order. The random order of task execution reflects, for instance, a situation where players and resources are the elements of an asynchronous distributed system, in which each process has its own independent clock ${ }^{2}$

In ROCGs, we consider a finite set of players, each having a unit length task that can be carried out by any element of a finite set of independent resources (machines). Each resource executes its assigned tasks in a randomly chosen order. As a result, a player may selfishly assign his task to several resources, hoping that his task will be completed in a short time by at least one of the resources. It is assumed that resource usage is costly; that is, every player has to pay for utilizing each of his chosen resources. More specifically, a player's aim is to minimize his expected total cost which is composed of the sum of the fixed costs over the set of his chosen resources and the cost of his task execution which is determined by the minimum completion time of his task by any of his chosen resources.

The presented model captures a very real issue that of redundant usage of resources, which is often the case in non-cooperative multi-agent systems, where selfish agents would try and run their jobs on several

\footnotetext{
${ }^{1}$ A short version (4 pages), titled "Asynchronous Congestion Games", appears in Proceedings of AAMAS-08 [15].

${ }^{2}$ The idea of using random ordering in order to reflect the asynchronous nature of processes in distributed systems is discussed, for example, in Monderer and Tennenholtz [11].
} 
resources in parallel, hoping that one would be faster. Therefore, providing theoretical analysis of these situations is of great importance. The model of ROCGs, perhaps, is one of the simplest to introduce the problem of wasteful usage of resources and asynchronous task execution into the context of congestion settings. One may think of a variety of generalizations of the model, some of which we mention in the concluding section of this paper. However, in these generalized models, most results would cease to hold, which motivates us to - first - study the relatively simple ROCG-model.

By considering the order of task execution, the study of ROCGs is related to the literature on selfish scheduling. There are two types of selfish scheduling: scheduling involving selfish machines [2, 6, 12, in which resources attempt to optimize their own objectives, and scheduling involving selfish tasks [1, 3, 7, in which each participant's objective is to minimize the completion time of his task. The latter type is closely related to congestion games.

Introducing a new class of games raises the important question of the existence of pure strategy equilibria as well as the computation of such equilibria. There are only few known classes of games which possess pure strategy equilibria, and there seems to be relatively little work providing efficient and exact algorithms for computing such equilibria. In this paper we introduce the class of ROCGs and prove that these games possess a Nash equilibrium in pure strategies, despite the non-existence of a potential function. In addition, we present a polynomial time algorithm for finding such an equilibrium in a given ROCG.

The rest of the paper is organized as follows. In Section 2 we define our model. In Sections 3,4 and 5 we present our results. In 3.1 we show that a $2 \times 2$ ROCG is a potential game. In 3.2 we observe that any ROCG with $n>2$ players or $m>2$ resources does not admit a potential function. In Section 4 we show that every ROCG possesses a pure strategy Nash equilibrium, despite the non-existence of a potential function. In Section 5 we present an $O\left(n m^{2}\right)$ algorithm for computing such an equilibrium. We prove our results in Section 6 , and conclude in Section 7.

2. The Model. Let $N=\{1, \ldots, n\}$ be a set of $n$ players and let $M=\left\{e_{1} \ldots, e_{m}\right\}$ be a set of $m$ resources. Player $i \in N$ chooses a strategy $\sigma_{i} \in \Sigma_{i}$ which is a nonempty subset of the resources: $\Sigma_{i}=P(M) \backslash\{\varnothing\}$. Given a subset $S \subseteq N$ of the players, the set of strategy combinations of the members of $S$ is denoted by $\Sigma_{S}=\times_{i \in S} \Sigma_{i}$, and the set of strategy combinations of the complement subset of players is denoted by $\Sigma_{-S}\left(\Sigma_{-S}=\Sigma_{N \backslash S}=\times_{i \in N \backslash S} \Sigma_{i}\right)$. For any $S \subseteq N$ and $\sigma=\left(\sigma_{i}\right)_{i \in S} \in \Sigma_{S}$, the (m-dimensional) congestion vector that corresponds to $S$ and $\sigma$ is $h(S, \sigma)=\left(h_{e}(S, \sigma)\right)_{e \in M}$, where $h_{e}(S, \sigma)=\left|\left\{i \in S: e \in \sigma_{i}\right\}\right|$. To simplify the notation, we denote the set of pure strategy profiles of all the players by $\Sigma\left(\Sigma=\Sigma_{N}\right)$; similarly, the congestion vector that corresponds to a "full" strategy profile $\sigma \in \Sigma$ is denoted by $h(\sigma)$.

The outcome for player $i \in N$ from $\sigma$ is the vector $x^{i}(\sigma)=\left(x_{e}^{i}(\sigma)\right)_{e \in M} \in\{1, \ldots, n, \infty\}^{m}$ of the ordering numbers of player $i$ 's task on all the resources, where $x_{e}^{i}(\sigma) \in\{1, \ldots, n\}$ for $e \in \sigma_{i}$ and $x_{e}^{i}(\sigma)=\infty$ for $e \notin \sigma_{i}$. The player's objective is to minimize his total cost that consists of the sum of the fixed costs over the set of resources he uses and the cost of the player's task execution. The fixed cost for utilizing each of the resources equals $t \geq 0$ units of money. The cost of task execution is a nonnegative, nondecreasing function of its completion time; thus, the longer it takes to complete the task execution, the greater is the cost incurred by the player. We assume that each player pays a fixed price, say $c$, for a unit of time his task is in the system before completed by at least one of the resources and, w.l.o.g., that this cost is one unit of money per unit of time. That is, the cost of a player's task execution is determined by the minimum among the completion times of his task by his chosen resources. Hence, the cost to player $i$ from a strategy profile $\sigma$ and his outcome $x^{i}(\sigma), c_{i}\left(\sigma, x^{i}(\sigma)\right)$, is defined as follows:

$$
c_{i}\left(\sigma, x^{i}(\sigma)\right)=\min _{e \in \sigma_{i}} x_{e}^{i}(\sigma)+\left|\sigma_{i}\right| t .
$$

Given a strategy profile $\sigma$, for any player $i \in N$ and resource $e \in \sigma_{i}$, let $X_{e}^{i}(\sigma)$ denote a random variable representing the ordering number of player $i$ 's task on resource $e$. Since it is assumed that each task requires a unit of time to be processed and each unit of time costs one unit of money, $X_{e}^{i}(\sigma)$ represents the cost to player $i$ for his task execution by resource $e$. We assume that $X_{e}^{i}(\sigma)$ is uniformly distributed over $\left\{1, \ldots, h_{e}(\sigma)\right\}$. The expected cost of player $i$ from strategy profile $\sigma, C_{i}(\sigma)$, is therefore:

$$
C_{i}(\sigma)=E\left(\min _{e \in \sigma_{i}} X_{e}^{i}(\sigma)\right)+\left|\sigma_{i}\right| t
$$




$$
\begin{aligned}
& =\sum_{q=1}^{\min _{e \in \sigma_{i}} h_{e}(\sigma)} \operatorname{Pr}\left(\min _{e \in \sigma_{i}} X_{e}^{i}(\sigma) \geq q\right)+\left|\sigma_{i}\right| t \\
& =\sum_{q=1}^{\min _{e \in \sigma_{i}} h_{e}(\sigma)} \prod_{e \in \sigma_{i}} \frac{h_{e}(\sigma)-q+1}{h_{e}(\sigma)}+\left|\sigma_{i}\right| t .
\end{aligned}
$$

The aim of each player is to minimize his own expected cost.

Note that if $t=0$ then the dominant strategy of each player is to assign his task to all of the resources. As a result, the system is overloaded and less efficient.

3. The (Non)-Existence of a Potential Function. Monderer and Shapley 10 introduced the notion of potential function (or, potential) as follows. Let $G$ be a game in strategic form with a finite set of players, $N$. The set of strategies of player $i \in N$ is $\Sigma_{i}$, and the payoff function of player $i$ is $C_{i}: \Sigma \rightarrow \mathbb{R}$, where $\Sigma=\times_{i \in N} \Sigma_{i}$ is the set of strategy profiles. A function $P: \Sigma \rightarrow \mathbb{R}$ is a potential function of $G$ if for every $i \in N$ and for every $\sigma_{-i} \in \Sigma_{-i}$,

$$
C_{i}\left(\sigma_{-i}, x\right)-C_{i}\left(\sigma_{-i}, y\right)=P\left(\sigma_{-i}, x\right)-P\left(\sigma_{-i}, y\right),
$$

for any $x, y \in \Sigma_{i}$. $G$ is called a potential game if it admits a potential function. The authors [10] showed that the classes of finite potential games and congestion games coincide.

In this section, we study the existence of a potential function in ROCGs. We show that a $2 \times 2$ ROCG is a potential game but any ROCG with $n>2$ players or $m>2$ resources does not possess a potential function. Hence, ROCGs are not congestion games.

3.1 ROCGs with 2 players and 2 resources. Here we present a potential function for an ROCG with 2 players and 2 resources. Let two players $N=\{1,2\}$ share a set of two resources $M=\left\{e_{1}, e_{2}\right\}$. In Table 1 we present the payoff matrix of the game. A potential function of the game is presented in Table 2. By exploring Tables 1 and 2, one can verify that for any two strategy profiles differing by the

\begin{tabular}{|c|c|c|c|}
\hline & $\left\{e_{1}\right\}$ & $\left\{e_{2}\right\}$ & $\left\{e_{1}, e_{2}\right\}$ \\
\hline$\left\{e_{1}\right\}$ & $C_{1}=\frac{3}{2}+t$ & $C_{1}=1+t$ & $C_{1}=\frac{3}{2}+t$ \\
& $C_{2}=\frac{3}{2}+t$ & $C_{2}=1+t$ & $C_{2}=1+2 t$ \\
\hline$\left\{e_{2}\right\}$ & $C_{1}=1+t$ & $C_{1}=\frac{3}{2}+t$ & $C_{1}=\frac{3}{2}+t$ \\
& $C_{2}=1+t$ & $C_{2}=\frac{3}{2}+t$ & $C_{2}=1+2 t$ \\
\hline$\left\{e_{1}, e_{2}\right\}$ & $C_{1}=1+2 t$ & $C_{1}=1+2 t$ & $C_{1}=\frac{5}{4}+2 t$ \\
& $C_{2}=\frac{3}{2}+t$ & $C_{2}=\frac{3}{2}+t$ & $C_{2}=\frac{5}{4}+2 t$ \\
\hline
\end{tabular}

Table 1: Players' payoffs in the $2 \times 2$ ROCG.

\begin{tabular}{|c|c|c|c|}
\hline & $\left\{e_{1}\right\}$ & $\left\{e_{2}\right\}$ & $\left\{e_{1}, e_{2}\right\}$ \\
\hline$\left\{e_{1}\right\}$ & $\frac{3}{4}$ & $\frac{1}{4}$ & $\frac{1}{4}+t$ \\
\hline$\left\{e_{2}\right\}$ & $\frac{1}{4}$ & $\frac{3}{4}$ & $\frac{1}{4}+t$ \\
\hline$\left\{e_{1}, e_{2}\right\}$ & $\frac{1}{4}+t$ & $\frac{1}{4}+t$ & $2 t$ \\
\hline
\end{tabular}

Table 2: A potential function of the $2 \times 2$ ROCG.

choice of a single player, the difference in the payoff of that player between the two profiles equals the corresponding increment in the function presented in Table 2. Therefore, this function is a potential.

3.2 ROCGs with $n>2$ players or $m>2$ resources. Here we show that any ROCG with $n>2$ players or $m>2$ resources does not admit a potential function. To prove this statement we use the following technical characterization of potential games.

Let $G$ be a game in strategic form with a set $N=\{1, \ldots, n\}$ of players, a set $\Sigma=\times_{i \in N} \Sigma_{i}$ of strategy profiles, and a vector $C=\left(C_{1}, \ldots, C_{n}\right)$ of payoff functions. A 4-cycle, i.e. a cycle of length 4 , in $\Sigma$ is a sequence $\tau=(\alpha \rightarrow \beta \rightarrow \gamma \rightarrow \delta \rightarrow \alpha)$ of strategy profiles, such that $\alpha=\left(x_{i}, x_{j}, z\right), \beta=\left(y_{i}, x_{j}, z\right)$, 
$\gamma=\left(y_{i}, y_{j}, z\right), \delta=\left(x_{i}, y_{j}, z\right)$, where $i, j \in N, x_{i}, y_{i} \in \Sigma_{i}, x_{j}, y_{j} \in \Sigma_{j}$, and $z \in \Sigma_{-\{i, j\}}$. A 4-cycle $\tau$ is zero-sum if

$$
\begin{aligned}
C(\tau)= & C_{i}(\alpha)-C_{i}(\beta)+C_{j}(\beta)-C_{j}(\gamma) \\
& +C_{i}(\gamma)-C_{i}(\delta)+C_{j}(\delta)-C_{j}(\alpha)=0,
\end{aligned}
$$

and non-zero-sum otherwise. Monderer and Shapley [10] showed that $G$ is a potential game if and only if it does not possess non-zero-sum 4-cycles.

Based on the above characterization, we show that every ROCG with $n>2$ players or $m>2$ resources does not admit a potential function. Let $G_{1}$ be any ROCG with $n>2$ players and $m \geq 2$ resources, and consider the 4-cycle $\tau_{1}$ which is formed by $\alpha=\left(\left\{e_{1}\right\},\left\{e_{2}\right\}, z\right), \beta=\left(\left\{e_{2}\right\},\left\{e_{2}\right\}, z\right), \gamma=\left(\left\{e_{2}\right\},\left\{e_{1}, e_{2}\right\}, z\right)$, $\delta=\left(\left\{e_{1}\right\},\left\{e_{1}, e_{2}\right\}, z\right)$, where $z \in \Sigma_{-\{1,2\}}$ satisfies $h_{e_{1}}(-\{1,2\}, z)<h_{e_{2}}(-\{1,2\}, z)$ (see Table 3). By

\begin{tabular}{|c|c|c|}
\hline & $\left\{e_{2}\right\}$ & \multicolumn{1}{c|}{$e_{1}, e_{2}$} \\
\hline$\left\{e_{1}\right\}$ & $C_{1}=\frac{h_{e_{1}}(-\{1,2\}, z)+2}{2}+t$ & $C_{1}=\frac{h_{e_{1}}(-\{1,2\}, z)+3}{2}+t$ \\
& $C_{2}=\frac{h_{e_{2}}(-\{1,2\}, z)+2}{2}+t$ & $C_{2}=\frac{h_{e_{1}}(-\{1,2\}, z)+3}{2}$ \\
& & $+\frac{1-\left(h_{e_{1}}(-\{1,2\}, z)+2\right)^{2}}{6\left(h_{e_{2}}(-\{1,2\}, z)+1\right)}+2 t$ \\
\hline \multirow{2}{*}{$\left.e_{2}\right\}$} & $C_{1}=\frac{h_{e_{2}}(-\{1,2\}, z)+3}{2}+t$ & $C_{1}=\frac{h_{e_{2}}(-\{1,2\}, z)+3}{2}+t$ \\
& $C_{2}=\frac{h_{e_{2}}(-\{1,2\}, z)+3}{2}+t$ & $C_{2}=\frac{h_{e_{1}}(-\{1,2\}, z)+2}{2}$ \\
& & $+\frac{1-\left(h_{e_{1}}(-\{1,2\}, z)+1\right)^{2}}{6\left(h_{e_{2}}(-\{1,2\}, z)+2\right)}+2 t$ \\
\hline
\end{tabular}

Table 3: Non-existence of potentials in ROCGs with $n>2$ players.

exploring Table 3 , one can verify that $C\left(\tau_{1}\right)$ is positive, which implies that $\tau_{1}$ is a non-zero-sum 4 -cycle. Hence, $G_{1}$ is not a potential game.

Now, let $G_{2}$ be any ROCG with $n=2$ players and $m>2$ resources. Consider the 4 -cycle $\tau_{2}$ which is formed by $\alpha=\left(\left\{e_{1}\right\},\left\{e_{3}\right\}\right), \beta=\left(\left\{e_{1}, e_{2}\right\},\left\{e_{3}\right\}\right), \gamma=\left(\left\{e_{1}, e_{2}\right\},\left\{e_{1}, e_{2}, e_{3}\right\}\right), \delta=\left(\left\{e_{1}\right\},\left\{e_{1}, e_{2}, e_{3}\right\}\right)$ (see Table 4).

\begin{tabular}{|c|c|c|}
\hline & $\left\{e_{3}\right\}$ & $\left\{e_{1}, e_{2}, e_{3}\right\}$ \\
\hline$\left\{e_{1}\right\}$ & $C_{1}=1+t$ & $C_{1}=\frac{3}{2}+t$ \\
& $C_{2}=1+t$ & $C_{2}=1+3 t$ \\
\hline$\left\{e_{1}, e_{2}\right\}$ & $C_{1}=1+2 t$ & $C_{1}=\frac{5}{4}+2 t$ \\
& $C_{2}=1+t$ & $C_{2}=1+3 t$ \\
\hline
\end{tabular}

Table 4: Non-existence of potentials in ROCGs with $m>2$ resources.

Direct calculation shows that $C\left(\tau_{2}\right)=-\frac{1}{4}$, implying that $\tau_{2}$ is a non-zero-sum 4-cycle and $G_{2}$ is not a potential game.

4. The Existence of a Pure Strategy Nash Equilibrium. Despite the fact that ROCGs, in general, are not potential games, in this section we prove that every ROCG possesses a Nash equilibrium in pure strategies.

One can easily verify that if the number of resources is greater than or equal to the number of players $(m \geq n)$ then the profile $\sigma=\left(e_{i}\right)_{i \in N}$ is a Nash equilibrium as well as an optimal strategy (one that minimizes the sum of the players' expected costs). However, if $m<n$, then proving the existence of such an equilibrium is not trivial, as is demonstrated below.

We start by showing that simple intuitive algorithms, like one presented in [13] for symmetric taxed congestion games with failures (STCGs), appear to be inapplicable in ROCGs. The STCG-algorithm proceeds as follows. It assigns the players to the resources by first assigning player 1 to resource $e_{1}$, then player 2 to resource $e_{2}$, and so on until player $m$ is assigned to the last resource $-e_{m}$. Then it continues with player $m+1$ going to resource $e_{1}$, and so on until player $n$ gets resource $e_{n \text { modm. }}$. A new sequence starts with player 1 assigned to resource $e_{n \operatorname{modm}+1}$ (or, to resource $e_{2}$, if $m$ divides $n$ ), and so on. The algorithm halts upon the first decline. Due to the resource and player symmetry of the ROCG-model, 
one may expect the above procedure to be valid for ROCGs as well. However, as the following example shows, this intuitive procedure does not work for ROCGs.

EXAMPLE 4.1 Consider an ROCG with 7 resources, $M=\left\{e_{1}, \ldots, e_{7}\right\}$, and 10 players, $N=$ $\{1,2, \ldots, 10\}$. The fixed cost for utilizing each of the resources is $t=\frac{199}{1200}$. The STCG-algorithm offers the players to apply resource additions in the following order:

\begin{tabular}{ccccccc}
$e_{1}$ & $e_{2}$ & $e_{3}$ & $e_{4}$ & $e_{5}$ & $e_{6}$ & $e_{7}$ \\
\hline \hline 1 & 2 & 3 & 4 & 5 & 6 & 7 \\
8 & 9 & 10 & 1 & 2 & 3 & 4 \\
5 & 6 & 7 & 8 & 9 & 10 & 1 \\
2 & 3 & 4 & 5 & 6 & 7 & 8 \\
9 & 10 & {$[1]$} & $2 \ldots$ & & &
\end{tabular}

For any strategy profile $\sigma \in \Sigma$, and a subset of resources $A \subseteq M$, let $h_{A}(\sigma)$ be a restriction of the congestion vector $h(\sigma)$ to $A$, and let $C\left(h_{A}(\sigma)\right)$ denote the expected cost of any player $i$ with $\sigma_{i}=A$. Thus,

$$
\begin{aligned}
& C(2)=\sum_{q=1}^{2} \frac{2-q+1}{2}+t=1+\frac{1}{2}+t=\frac{3}{2}+t=\frac{1999}{1200} \\
& C(2,2)=\sum_{q=1}^{2}\left(\frac{2-q+1}{2}\right)^{2}+2 t=1+\left(\frac{1}{2}\right)^{2}+2 t=\frac{5}{4}+2 t=\frac{1898}{1200} \\
& C(3,2)=\sum_{q=1}^{2} \frac{3-q+1}{3} \cdot \frac{2-q+1}{2}+2 t=1+\frac{1}{2} \cdot \frac{2}{3}+2 t=\frac{4}{3}+2 t=\frac{1998}{1200}
\end{aligned}
$$

Since $C(2,2)<C(3,2)<C(2)$, the offers proposed by the algorithm at steps 11-17 (see rows 2 and 3 ) will be accepted. Furthermore,

$$
\begin{aligned}
& C(3)=\sum_{q=1}^{3} \frac{3-q+1}{3}+t=1+\frac{2}{3}+\frac{1}{3}+t=2+t=\frac{2599}{1200}=\frac{7797}{3600}> \\
& C(3,3)=\sum_{q=1}^{3}\left(\frac{3-q+1}{3}\right)^{2}+2 t=1+\left(\frac{2}{3}\right)^{2}+\left(\frac{1}{3}\right)^{2}+2 t=\frac{14}{9}+2 t=\frac{3397}{1800}=\frac{6794}{3600}> \\
& C(3,3,3)=\sum_{q=1}^{3}\left(\frac{3-q+1}{3}\right)^{3}+3 t=1+\left(\frac{2}{3}\right)^{3}+\left(\frac{1}{3}\right)^{3}+3 t=\frac{13}{9}+2 t=\frac{2197}{1200}=\frac{6591}{3600}
\end{aligned}
$$

imply additions at steps 18 -21 (row 3). Similarly, one can show that $C(4,3,3)<C(3,3), C(4,4,3)<$ $C(4,3)$ and $C(4,4,4)<C(4,4)$, implying additions at steps 22-27 (row 4).

Consider now row 5. Players 9 and 10 will add resources $e_{1}$ and $e_{2}$, respectively, as $C(5,4,4)<C(4,4)$. At the following step the algorithm will receive decline from player 1 who will refuse to add resource $e_{3}$. This is since $C(5,5,4,4)>C(5,4,4)$ (follows from $t=\frac{199}{1200}>\frac{33}{200}$ ). However, $t=\frac{199}{1200}<\frac{24}{125}$ yields $C(5,5,5,4)<C(5,5,4)$, implying that the resulting strategy profile is not in equilibrium, as player 2 wishes to add a resource (any of $e_{3}-e_{7}$, excluded $e_{5}$ ). Note that player 1 uses two resources with the congestion of 4 and one resource with the congestion of 5, and in this situation has no incentive to add one more resource as the marginal contribution of this operation will not cover the fixed cost for adding the resource. In contrast, player 2 uses two resources with the congestion of 5 and one resource with the congestion of 4, and hence his expected completion time is higher than that of player 1, implying larger marginal contribution from adding a resource, which also turns out to be sufficient to cover the fixed cost.

We now proceed and present our proof. It uses the notion of stability under single moves, previously presented in [14, and proceeds as follows. Below, in 4.1 we define three types of single moves (A-, D- and S-moves) and show that a profile which is stable under all these moves is a Nash equilibrium (see Lemma 4.1. In 4.2 we observe that the DS-stabl ${ }^{3}$ profile is easy to find, but the existence of a profile which is

\footnotetext{
${ }^{3}$ A strategy profile which is stable under D- and S-moves (see Definition 4.2 .
} 
stable under all three types of single moves is not obvious (see Lemma 4.2 and the discussion following it). We look for such a profile using two types of addition operations, which are defined in 4.3. Lemma 4.3 in this subsection describes how these additions affect DS-stable profiles. Based on this lemma, in 4.4 we prove that for some DS-stable profiles the above additions do not ruin the DS-stability (see Lemma 4.4). We complete our proof by showing that applying a finite series of addition operations to such a profile results in an equilibrium (see Lemma 4.5 and Corollary 4.3. The formal proofs of all the lemmas are presented in Section 6 .

4.1 The single profitable move property. As pointed out in [14], in a congestion setting, we are mainly interested in three types of single moves, where each type is a deviation involving a single resource, as follows.

Definition 4.1 [14] For any strategy profile $\sigma \in \Sigma$ and for any player $i \in N$, the operation of adding precisely one resource to his strategy, $\sigma_{i}$, is called an $\boldsymbol{A}$-move of $i$ from $\sigma$. Similarly, the operation of dropping a single resource is called a D-move, and the operation of switching one resource with another is called an $\boldsymbol{S}$-move.

The following observation provides technical characterizations of single moves and is heavily utilized in the presentation and the proofs of our results.

Observation 4.1 Given a profile $\sigma$, let $h$ denote its corresponding congestion vector $(h=h(\sigma))$, and assume there exist $a, b \in M$ and $i \in N$ such that $a \in \sigma_{i}$ and $b \notin \sigma_{i}$. Then,

(1) If a D-move with a is profitable for $i$ then

$$
t>\sum_{q=1}^{\min _{e \in \sigma_{i}-a} h_{e}}\left(\prod_{e \in \sigma_{i}-a} \frac{h_{e}-q+1}{h_{e}}\right) \frac{q-1}{h_{a}} .
$$

If $\min _{e \in \sigma_{i}-a} h_{e}=\min _{e \in \sigma_{i}} h_{e}$ and the D-move with a is non-profitable for $i$ then

$$
t \leq \sum_{q=1}^{\min _{e \in \sigma_{i}-a} h_{e}}\left(\prod_{e \in \sigma_{i}-a} \frac{h_{e}-q+1}{h_{e}}\right) \frac{q-1}{h_{a}} .
$$

(2) If an A-move with $b$ is non-profitable for $i$ then

$$
t \geq \sum_{q=1}^{\min _{e \in \sigma_{i}} h_{e}}\left(\prod_{e \in \sigma_{i}} \frac{h_{e}-q+1}{h_{e}}\right) \frac{q-1}{h_{b}+1} .
$$

If $\exists e \in \sigma_{i}$ such that $h_{e} \leq h_{b}+1$ and the A-move with $b$ is profitable for $i$ then

$$
t<\sum_{q=1}^{\min _{e \in \sigma_{i}} h_{e}}\left(\prod_{e \in \sigma_{i}} \frac{h_{e}-q+1}{h_{e}}\right) \frac{q-1}{h_{b}+1}
$$

Note that if $\min _{e \in \sigma_{i}} h_{e}=1$ then there exists resource $e \in \sigma_{i}$ such that player $i$ is its only user, and $C_{i}(\sigma)=1+\left|\sigma_{i}\right| t$. Therefore, $i$ cannot reduce his cost by applying an A-move.

(3) An S-move from a to $b$ is profitable for $i$ if and only if $h_{b}+1<h_{a}$.

Lemma 4.1 below implies that any strategy profile in which no player wishes unilaterally to apply a single A-, D- or S-move, is a Nash equilibrium. This property is called the single profitable move property and it allows us to consider only single moves rather than considering all possible deviations.

LEMMA 4.1 (The single profitable move property) Given an ROCG, let $\sigma \in \Sigma$ be a strategy profile which is not in equilibrium, and let $i \in N$ be a player for which a profitable deviation from $\sigma$ is available. Then, $i$ has a profitable $A$-, $D$ - or $S$-move from $\sigma$. 
4.2 Stability under single moves. By Lemma 4.1. in order to prove the existence of a pure strategy Nash equilibrium in games possessing the single profitable move property, it suffices to present a strategy profile for which no player wishes to unilaterally apply an A-, D- or S-move. This observation motivates the following definition.

Definition 4.2 [14] A strategy profile $\sigma$ is said to be $\boldsymbol{A}$-stable (resp., D-stable, S-stable) if there are no players with a profitable $A$ - (resp., D-, S-) move from $\sigma$. An $A$ - and D-stable profile (resp., A- and S-stable, D- and S-stable) will be termed AD-stable (resp., AS-stable, DS-stable).

In order to investigate stability under single moves in ROCGs we use the notions of light and heavy resources as well as of even and nearly-even strategy profiles.

Definition 4.3 [14] Given a strategy profile $\sigma$, resource $e^{\prime}$ is called $\sigma$-light if $e^{\prime} \in \arg _{\min } \in M h_{e}(\sigma)$ and $\sigma$-heavy otherwise. A strategy profile $\sigma$ with no heavy resources will be termed even. An even strategy profile with a common congestion of $k$ on the resources will be termed $\boldsymbol{k}$-even. A strategy profile $\sigma$ satisfying $\left|h_{e}(\sigma)-h_{e^{\prime}}(\sigma)\right| \leq 1$ for all $e, e^{\prime} \in M$ will be termed nearly-even.

Obviously, every even strategy profile is nearly-even. In addition, in a nearly-even strategy profile all heavy resources (if such exist) have the same congestion. Moreover, as is shown in the following lemma, the notions of nearly-evenness and S-stability are strongly connected.

LEMMA 4.2 In an ROCG, a strategy profile is S-stable if and only if it is nearly-even.

Note that the pairwise intersections of the set of S-stable strategy profiles with the set of A-stable profiles or the set of D-stable profiles are not empty. In particular, the strategy profile $\sigma^{M}=(M, \ldots, M)$ is AS-stable, while the profile $\sigma^{0}=\left(e_{i \bmod m+1}\right)_{i \in N}$ is DS-stable. However, at first glance, it is not clear whether there exists a profile which is stable under all three types of single moves, or even if there is an AD-stable profile.

Intuitively, one can try to achieve a Nash equilibrium by selecting a profile which is stable under two types of single moves and applying on it a series of single moves of the third type. For instance, one can pick a DS-stable strategy profile and try to transform it into a Nash equilibrium by applying on it a series of profitable A-moves. However, such moves may destroy the D- or the S-stability of the selected profile; moreover, an A-move from the selected profile may initiate a long chain of D- and S-moves (see Example 4.2 in the sequel). Therefore, the chosen actions have to be picked out in a careful and subtle way. In this context, we first restrict the set of available A-moves to the subset of one- and two-step addition operations, as defined in the sequel.

4.3 One- and two-step additions. Let $\sigma \in \Sigma$ be a strategy profile and let $h$ denote its corresponding congestion vector $(h=h(\sigma))$. For each player $i \in N$, let $e^{i} \in \arg \min _{e \in M \backslash \sigma_{i}} h_{e}$. That is, $e^{i}$ is a lightest resource not previously chosen by $i$. Then, one can make the following (straightforward) observation.

Observation 4.2 If there exists a profitable A-move for player $i$, then an A-move with $e^{i}$, a lightest resource not chosen previously by $i$, is profitable for $i$ as well.

If no player wishes to change his strategy in this manner, i.e. $C_{i}(\sigma) \leq C_{i}\left(\sigma_{-i}, \sigma_{i}+e^{i}\right)$ for all $i \in N$, then by Observation 4.2 $C_{i}(\sigma) \leq C_{i}\left(\sigma_{-i}, \sigma_{i}+a\right)$ for all $i \in N$ and $a \in M \backslash \sigma_{i}$. Hence, $\sigma$ is A-stable. Otherwise, let $N(\sigma)$ denote the subset of all players for which there exists $e^{i}$ such that a unilateral addition of $e^{i}$ is profitable. Let $a \in \arg \min _{e^{i}: i \in N(\sigma)} h_{e^{i}}$. Let also $i \in N(\sigma)$ be the player for which $e^{i}=a$. If $a$ is $\sigma$-light, then let $\sigma^{\prime}=\left(\sigma_{-i}, \sigma_{i}+a\right)$. In this case we say that $\sigma^{\prime}$ is obtained from $\sigma$ by ane-step addition of resource $a$, and $a$ is called an added resource. If $a$ is $\sigma$-heavy then there exists a $\sigma$-light resource $b$ and a player $j$ such that $a \in \sigma_{j}$ and $b \notin \sigma_{j}$. Then let $\sigma^{\prime}=\left(\sigma_{-\{i, j\}}, \sigma_{i}+a, \sigma_{j}-a+b\right)$. In this case we say that $\sigma^{\prime}$ is obtained from $\sigma$ by a two-step addition of resource $b$, and $b$ is called an added resource.

We notice that, in both cases, the congestion of each resource in $\sigma^{\prime}$ is the same as in $\sigma$, except for the added resource, with the congestion in $\sigma^{\prime}$ increased by 1 . Thus, if $\sigma$ is nearly-even then $\sigma^{\prime}$ is also 
nearly-even (since the added resource is $\sigma$-light). Then, Lemma 4.2 implies the S-stability of $\sigma^{\prime}$. Lemma 4.3 below shows that if, in addition, $\sigma$ is D-stable then the only potential cause for the non-D-stability of $\sigma^{\prime}$ is the existence of player $i \in N$ with $\sigma_{i}^{\prime}=\sigma_{i}$ who wishes to drop the added resource $a$.

LEMMA 4.3 Let $\sigma$ be a nearly-even and D-stable strategy profile of a given ROCG, and let $\sigma^{\prime}$ be obtained from $\sigma$ by a one- or two-step addition of resource a. Then, there are no profitable D-moves for any player $i \in N$ with $\sigma_{i}^{\prime} \neq \sigma_{i}$. For $i \in N$ with $\sigma_{i}^{\prime}=\sigma_{i}$, the only possible profitable D-move (if such exists) is to drop the added resource $a$.

Note that although we did not succeed in keeping the D-stability, we have significantly reduced the set of possible post-addition D-moves. However, as we show in the following example, we still may encounter a situation in which a D-move with the added resources causes a long sequence of (profitable) D-moves from a current profile.

EXAMPLE 4.2 Consider an ROCG with 17 players, $N=\{1,2, \ldots, 17\}$, and 7 resources, $M=$ $\left\{e_{1}, \ldots, e_{7}\right\}$. The fixed cost for utilizing each of the resources is given by $t=\frac{3}{8}$. Let $\sigma$ be a strategy profile in which the players are allocated to the resources in the following way:

\begin{tabular}{ccccccc}
$e_{1}$ & $e_{2}$ & $e_{3}$ & $e_{4}$ & $e_{5}$ & $e_{6}$ & $e_{7}$ \\
\hline \hline 1 & 2 & 7 & 8 & 1 & 2 & 14 \\
3 & 4 & 9 & 10 & 3 & 4 & 15 \\
5 & 6 & 11 & 12 & 5 & 6 & 16 \\
& & 13 & 13 & & & 17
\end{tabular}

Note that $h_{e_{1}}(\sigma)=h_{e_{2}}(\sigma)=h_{e_{5}}(\sigma)=h_{e_{6}}(\sigma)=3$ and $h_{e_{3}}(\sigma)=h_{e_{4}}(\sigma)=h_{e_{7}}(\sigma)=4$; that is, $\sigma$ is nearly-even and therefore $S$-stable (by Lemma 4.2). In addition, as we show below, no profitable D-moves from $\sigma$ are available.

For any subset of resources, $A \subseteq M$, let $C\left(h_{A}(\sigma)\right)$, where $h_{A}(\sigma)$ is a restriction of the congestion vector $h(\sigma)$ to $A$, denote the expected cost of player $i$ with $\sigma_{i}=A$. Thus,

$$
\begin{aligned}
& C(3)=\sum_{q=1}^{3} \frac{3-q+1}{3}+t=1+\frac{2}{3}+\frac{1}{3}+t=2+t=\frac{19}{8}=\frac{171}{72} \\
& C(4)=\sum_{q=1}^{4} \frac{4-q+1}{4}+t=1+\frac{3}{4}+\frac{1}{2}+\frac{1}{4}+t=\frac{5}{2}+t=\frac{23}{8}=\frac{207}{72} \\
& C(3,3)=\sum_{q=1}^{3}\left(\frac{3-q+1}{3}\right)^{2}+2 t=1+\left(\frac{2}{3}\right)^{2}+\left(\frac{1}{3}\right)^{2}+2 t=\frac{13}{9}+2 t=\frac{79}{36}=\frac{158}{72} \\
& C(3,4)=\sum_{q=1}^{3} \frac{3-q+1}{3} \cdot \frac{4-q+1}{4}+2 t=1+\frac{2}{3} \cdot \frac{3}{4}+\frac{1}{3} \cdot \frac{1}{2}+2 t=\frac{5}{3}+2 t=\frac{29}{12}=\frac{174}{72} \\
& C(4,4)=\sum_{q=1}^{4}\left(\frac{4-q+1}{4}\right)^{2}+2 t=1+\left(\frac{3}{4}\right)^{2}+\left(\frac{1}{2}\right)^{2}+\left(\frac{1}{4}\right)^{2}+2 t=\frac{15}{8}+2 t=\frac{21}{8}=\frac{189}{72}
\end{aligned}
$$

The expected cost to each of the players $1-6$ is $C(3,3)=\frac{158}{72}<\frac{171}{72}=C(3)$, and player 13 's cost is $C(4,4)=\frac{189}{72}<\frac{207}{72}=C(4)$. This implies that none of the above players is interested in applying a $D$-move with one of his resources. The other players are using a single resource each, hence no D-moves are available to these players as well. Therefore, $\sigma$ is a DS-stable profile.

Now, since $C(4,4)<C(4)$, it follows that player 7 wishes to apply an A-move with any of the resources $e_{1}, e_{2}, e_{5}, e_{6}$. Let $\sigma^{\mathrm{i}}=\left(\sigma_{7} \cup\left\{e_{1}\right\}, \sigma_{-7}\right)$ (note that $e_{1}$ is $\sigma$-light, i.e. $\sigma^{\mathrm{i}}$ is obtained from $\sigma$ by the one-step addition of $e_{1}$ by player 7$)$ as presented below:

\begin{tabular}{ccccccc}
$e_{1}$ & $e_{2}$ & $e_{3}$ & $e_{4}$ & $e_{5}$ & $e_{6}$ & $e_{7}$ \\
\hline \hline 1 & 2 & 7 & 8 & 1 & 2 & 14 \\
3 & 4 & 9 & 10 & 3 & 4 & 15 \\
5 & 6 & 11 & 12 & 5 & 6 & 16 \\
7 & & 13 & 13 & & & 17
\end{tabular}


Notice that since $C(3,4)=\frac{174}{72}>\frac{171}{72}=C(3)$, the profile $\sigma^{\mathrm{i}}$ is not D-stable, and each of players player $1,3,5$ wishes to apply a D-move from the added resource, $e_{1}$ (by Lemma 4.3, this is the only possible profitable D-move). Let $\sigma^{\mathrm{ii}}=\left(\sigma_{5} \backslash\left\{e_{1}\right\}, \sigma_{-5}\right)$ :

\begin{tabular}{ccccccc}
$e_{1}$ & $e_{2}$ & $e_{3}$ & $e_{4}$ & $e_{5}$ & $e_{6}$ & $e_{7}$ \\
\hline \hline 1 & 2 & 7 & 8 & 1 & 2 & 14 \\
3 & 4 & 9 & 10 & 3 & 4 & 15 \\
7 & 6 & 11 & 12 & 5 & 6 & 16 \\
& & 13 & 13 & & & 17
\end{tabular}

Observe that profile $\sigma^{\mathrm{ii}}$ is not D-stable as well, since player 7 wishes to drop resource $e_{3}$, and let $\sigma^{\mathrm{iii}}=$ $\left(\sigma_{7} \backslash\left\{e_{3}\right\}, \sigma_{-7}\right)$ :

\begin{tabular}{ccccccc}
$e_{1}$ & $e_{2}$ & $e_{3}$ & $e_{4}$ & $e_{5}$ & $e_{6}$ & $e_{7}$ \\
\hline \hline 1 & 2 & 9 & 8 & 1 & 2 & 14 \\
3 & 4 & 11 & 10 & 3 & 4 & 15 \\
7 & 6 & 13 & 12 & 5 & 6 & 16 \\
& & & 13 & & & 17
\end{tabular}

Note that the moves of player 7 demonstrate the following chain of preferences: utilizing 2 resources of congestion 4 is preferred by using 1 resource of congestion 4 (his A-move operation); now, after the D-move of player 5, using 1 resource of congestion 3 is preferred by using 1 resource of congestion 3 and 1 resource of congestion 4 (the D-move of player 7 ).

Profile $\sigma^{\mathrm{iii}}$ is not in-D-stable, too - the D-move with $e_{4}$ is profitable for player 13 (for the same reason as previously for players 5 and 7$)$, and let $\sigma^{\mathrm{iv}}=\left(\sigma_{13} \backslash\left\{e_{4}\right\}, \sigma_{-13}\right)$ :

\begin{tabular}{ccccccc}
$e_{1}$ & $e_{2}$ & $e_{3}$ & $e_{4}$ & $e_{5}$ & $e_{6}$ & $e_{7}$ \\
\hline \hline 1 & 2 & 9 & 8 & 1 & 2 & 14 \\
3 & 4 & 11 & 10 & 3 & 4 & 15 \\
7 & 6 & 13 & 12 & 5 & 6 & 16 \\
& & & & & & 17
\end{tabular}

Although the resulting profile, $\sigma^{\mathrm{iv}}$, is DS-stable (no more profitable D-moves are available), it is not a Nash equilibrium, since any of the players 14 - 17 would now benefit from an A-move with any of the resources in $M \backslash\left\{e_{7}\right\}$ (since $C(4,4)<C(4)$ ), and a new chain of moves will begin.

As one may learn from the above example, the addition of a resource $a$ by player $i$ may force player $j$ to drop the added resource, $a$. This, in turn, may cause player $i$ to drop another resource, say $b$. This is since the D-move of $j$ from $a$ has decreased the congestion of $a$; hence, the $i$ 's chance to get his task executed earlier by a ( $\sigma$-heavy) resource $b$ has been decreased. For the same reason, the D-move of $i$ from $b$ may cause a chain of D-moves from other heavy resources by different players. Although the length of such a chain is bounded by $m$, the number of resources, it is not clear whether the one-/two-step addition dynamics converges to an equilibrium if it initializes with an arbitrary DS-stable profile. This motivates us to present the term of post-addition D-stability which plays a central role in our method, as follows.

4.4 Post-addition D-stability. Let $\sigma \in \Sigma$ be a strategy profile and let $\sigma^{\prime}$ be obtained from $\sigma$ by applying a one- or two-step addition operation. Then, based on Lemma 4.3, $\sigma$ is said to be post-addition $D$-stable if $\sigma^{\prime}$ does not admit profitable D-moves with the added resource. Formally, the post-addition D-stability is defined as follows.

Definition 4.4 A strategy profile $\sigma$ of a given ROCG is called post-addition D-stable if

$$
t \leq \sum_{q=1}^{\min _{e \in \sigma_{i}-a} h_{e}(\sigma)}\left(\prod_{e \in \sigma_{i}-a} \frac{h_{e}(\sigma)-q+1}{h_{e}(\sigma)}\right) \frac{q-1}{h_{a}(\sigma)+1},
$$

for every $i \in N$ with $\left|\sigma_{i}\right|>1$ and for every $\sigma$-light resource $a \in \sigma_{i}$.

We note that by Observation 4.1, inequality (1) implies the non-profitability of a D-move with the added resource. 
Let $\Sigma^{0} \subseteq \Sigma$ denote the subset of all D-stable strategy profiles, and let $\Sigma^{1} \subseteq \Sigma^{0}$ be the subset of all even and D-stable strategy profiles. By Lemma 4.2 every profile in $\Sigma^{1}$ (if such exists) is S-stable. For any $\sigma \in \Sigma^{1}$, let $H^{\sigma}$ denote the common congestion on the resources, and let $\Sigma^{2} \subseteq \Sigma^{1}$ be the subset of $\Sigma^{1}$ consisting of all those profiles with maximum congestion on the resources: $\Sigma^{2}=\arg \max _{\sigma \in \Sigma^{1}} H^{\sigma}$. Then,

Lemma 4.4 Given an ROCG, there exists a strategy profile $\sigma \in \Sigma^{2}$ that is either a pure strategy Nash equilibrium or post-addition D-stable.

It is not clear, by first look, if the existence of a post-addition D-stable strategy profile implies the existence of a pure strategy Nash equilibrium. To show such an implication, post-addition D-stability should be preserved while applying a series of addition operations. In addition, such a series of addition operations should converge to a pure strategy Nash equilibrium in a finite number of steps. In this context, Lemma 4.5 and Corollary 4.3 below provide the needed steps for completing the proof of existence of a pure strategy equilibrium.

Lemma 4.5 Given an ROCG, let $\sigma$ be a nearly-even and post-addition D-stable strategy profile, and let $\sigma^{\prime}$ be obtained from $\sigma$ by applying on it a one- or two-step addition operation. If $\min _{e \in M} h_{e}\left(\sigma^{\prime}\right)=$ $\min _{e \in M} h_{e}(\sigma)$ then $\sigma^{\prime}$ is also nearly-even and post-addition D-stable.

Corollary 4.3 By Lemma 4.5, if we can find a post-addition D-stable strategy profile $\sigma^{\prime}$ that lies in $\Sigma^{2}$, then a pure strategy Nash equilibrium can be achieved by applying on $\sigma^{\prime}$, in a sequential manner, less than $m$ one-/two-step addition operations. This is because if we perform $m$ addition operations then an even D-stable strategy profile $\sigma^{\prime \prime}$ with $H^{\sigma^{\prime \prime}}>H^{\sigma^{\prime}}$ is obtained, contradicting $\sigma^{\prime} \in \Sigma^{2}$.

Theorem 4.4 below follows directly from Lemmas 4.4 and 4.5 , and Corollary 4.3 .

THEOREM 4.4 Every ROCG possesses a Nash equilibrium in pure strategies.

5. Computation of a Pure Strategy Nash Equilibrium. We are now ready to present our Random Order Nash Equilibrium (RONE)-algorithm that constructs a pure strategy Nash equilibrium in any given ROCG. Let us start with a brief description of the algorithm:

- Based on Lemma 4.5 the goal of the algorithm is to find a strategy profile in $\Sigma^{2}$ which is either a pure strategy Nash equilibrium or post-addition D-stable. In the latter case, the strategy profile can be turned into a Nash equilibrium by applying on it at most $m-1$ one-/two-step addition operations. For that, the algorithm has to determine a value $k^{*}=\max _{\sigma \in \Sigma^{1}} H^{\sigma}$ that represents the common congestion on the resources for any strategy profile in $\Sigma^{2}$.

- To find $k^{*}$ as above, the algorithm uses a variable $k$ initiated with the value $k=n$ and gradually decreases until $k^{*}$ is found (Steps [0]- [1]).

- For $k=n$, the only even strategy profile with $n$ being its common congestion is $\sigma=(M, \ldots, M)$, which is obviously A- and S-stable. If $\sigma$ is also D-stable then $k^{*}=n$, and the algorithm outputs $\sigma$ and halts (Step [0]). Otherwise, $k^{*}<n$ and the algorithm proceeds with $k=n-1$ (Step [1]).

- Given $\left\lfloor\frac{n}{m}\right\rfloor<k<n$, the algorithm checks whether a $k$-even D-stable strategy profile exists. If there is no such profile then $k^{*}<k$ and the algorithm proceeds with the next value of $k$ (repeating Step [1]). Otherwise, $k^{*}=k$.

- If $k^{*}=\left\lfloor\frac{n}{m}\right\rfloor$ then the algorithm constructs a strategy profile $\sigma=\left(e_{i \bmod m}\right)_{i \in N}$ (Step [2]). As we show in the proof of Theorem 5.1, $\sigma$ is a Nash equilibrium.

- Otherwise, $k^{*}>\left\lfloor\frac{n}{m}\right\rfloor$. In this case, the algorithm constructs a $k^{*}$-even strategy profile $\sigma$ with $n^{*}=n\left(\left\lfloor\frac{k^{*} m}{n}\right\rfloor+1\right)-k^{*} m$ players using $\left\lfloor\frac{k^{*} m}{n}\right\rfloor$ resources and $n-n^{*}=k^{*} m-n\left\lfloor\frac{k^{*} m}{n}\right\rfloor$ players using $\left\lfloor\frac{k^{*} m}{n}\right\rfloor+1$ resources (Step [3]). As we show in the proof of Theorem 5.1, the obtained $\sigma$ is D- and S-stable. If $\sigma$ is also A-stable then the algorithm outputs $\sigma$ and halts (Step [4]). Otherwise, we show that $\sigma \in \Sigma^{2}$ and is post-addition D-stable. Then, based on Lemma 4.5 and Corollary 4.3 , a pure strategy Nash equilibrium is achieved by applying at most $m-1$ one- or two-step additions on $\sigma$ (Steps [5] - [9]). 
The RONE-algorithm is presented below.

\section{RONE-algorithm}

Step [0] If $t \leq \sum_{q=1}^{n}\left(\frac{n-q+1}{n}\right)^{m-1} \frac{q-1}{n}$ then set $\sigma:=(M, \ldots, M)$ and QUIT;

Otherwise, set $k:=n-1$ and go to Step [1];

Step [1] If $t>\sum_{q=1}^{k}\left(\frac{k-q+1}{k}\right)^{\left\lceil\frac{k m}{n}\right\rceil-1} \frac{q-1}{k}$ then set $k:=k-1$; Otherwise go to Step [3];

Step [2] If $k=\left\lfloor\frac{n}{m}\right\rfloor$ then set $\sigma:=\left(e_{i \bmod m}\right)_{i \in N}$ and QUIT; Otherwise go to Step [1];

Step [3] Set $n^{*}:=n\left(\left\lfloor\frac{k m}{n}\right\rfloor+1\right)-k m$;

For $i=1$ to $n^{*}$ :

Set $\sigma_{i}=\left\{e_{r} \in M: 1 \leq r \leq\left\lfloor\frac{k m}{n}\right\rfloor\right\}$

and reorder the resources:

for all $e_{r} \in M$ set $e_{r}:=e_{\left(r+\left\lfloor\frac{k m}{n}\right\rfloor\right) \bmod m}$;

If $n^{*}=n$ then go to Step [4];

Otherwise, for $i=n^{*}+1$ to $n$ :

Set $\sigma_{i}=\left\{e_{r} \in M: 1 \leq r \leq\left\lfloor\frac{k m}{n}\right\rfloor+1\right\}$

and reorder the resources:

for all $e_{r} \in M$ set $e_{r}:=e_{\left(r+\left\lfloor\frac{k m}{n}\right\rfloor+1\right) \bmod m}$;

Step [4] If $t \geq \sum_{q=1}^{k}\left(\frac{k-q+1}{k}\right)^{\left\lfloor\frac{k m}{n}\right\rfloor} \frac{q-1}{k+1}$ then QUIT;

Step [5] For all $i \in N$, select $e^{i} \in \arg \min _{e \in M \backslash \sigma_{i}} h_{e}(\sigma)$;

Step [6] Set $N(\sigma):=\left\{i \in N: C_{i}\left(\sigma_{-i}, \sigma_{i}+e^{i}\right)<C_{i}(\sigma)\right\}$; If $N(\sigma)=\varnothing$ then QUIT;

Step [7] Set $M(\sigma):=\left\{e \in M: \exists i \in N(\sigma), e=e^{i}\right\}$;

Step [8] Select $a^{*} \in \arg \min _{e \in M(\sigma)} h_{e}(\sigma)$ and $i^{*} \in\left\{i \in N(\sigma): e^{i}=a^{*}\right\}$;

Step [9] If $a^{*}$ is $\sigma$-light set $\sigma_{i^{*}}:=\sigma_{i^{*}}+a^{*}$ and go to Step [5];

Otherwise select a $\sigma$-light resource $b^{*}$ and $j^{*} \in\left\{i \in N: a^{*} \in \sigma_{i}, b^{*} \notin \sigma_{i}\right\}$, set $\sigma_{i^{*}}:=\sigma_{i^{*}}+a^{*}, \sigma_{j^{*}}:=\sigma_{j^{*}}-a^{*}+b^{*}$, and go to Step [5].

THEOREM 5.1 The RONE-algorithm finds a pure strategy Nash equilibrium in any given ROCG, and its time complexity is $O\left(\mathrm{~nm}^{2}\right)$.

6. Proofs. Here we provide the proofs of our results. We start by making some technical observations that will help us in their presentation. Let $\sigma \in \Sigma$ be a strategy profile and let $h$ denote its corresponding congestion vector $(h=h(\sigma))$. Let $i \in N$ and $a \in \sigma_{i}$. If a D-move with $a$ is profitable for $i$ then $C_{i}\left(\sigma_{-i}, \sigma_{i}-a\right)<C_{i}(\sigma)$. That is,

$$
\begin{aligned}
\sum_{q=1}^{\min _{e \in \sigma_{i}-a} h_{e}} \prod_{e \in \sigma_{i}-a} \frac{h_{e}-q+1}{h_{e}}+\left(\left|\sigma_{i}\right|-1\right) t<\sum_{q=1}^{\min _{e \in \sigma_{i}} h_{e}} \prod_{e \in \sigma_{i}} \frac{h_{e}-q+1}{h_{e}}+\left|\sigma_{i}\right| t \\
\Leftrightarrow t>\sum_{q=1}^{\min _{e \in \sigma_{i}-a} h_{e}} \prod_{e \in \sigma_{i}-a} \frac{h_{e}-q+1}{h_{e}}-\sum_{q=1}^{\min _{e \in \sigma_{i}}} \prod_{e \in \sigma_{i}} \frac{h_{e}-q+1}{h_{e}} .
\end{aligned}
$$

Since $\min _{e \in \sigma_{i}-a} h_{e} \geq \min _{e \in \sigma_{i}} h_{e}$, the above implies

$$
t>\sum_{q=1}^{\min _{e \in \sigma_{i}-a} h_{e}}\left(\prod_{e \in \sigma_{i}-a} \frac{h_{e}-q+1}{h_{e}}\right) \frac{q-1}{h_{a}} \geq \sum_{q=1}^{\min _{e \in \sigma_{i}} h_{e}}\left(\prod_{e \in \sigma_{i}-a} \frac{h_{e}-q+1}{h_{e}}\right) \frac{q-1}{h_{a}} .
$$


If the D-move with $a$ is non-profitable for $i$ then $C_{i}(\sigma) \leq C_{i}\left(\sigma_{-i}, \sigma_{i}-a\right)$ and if the move is strictly non-profitable then the inequality is strict. Hence, if the D-move with $a$ is non-profitable for $i$ then

$$
t \leq \sum_{q=1}^{\min _{e \in \sigma_{i}-a} h_{e}} \prod_{e \in \sigma_{i}-a} \frac{h_{e}-q+1}{h_{e}}-\sum_{q=1}^{\min _{e} \sigma_{i}} \prod_{e \in \sigma_{i}} \frac{h_{e}-q+1}{h_{e}} .
$$

We also notice that if $\min _{e \in \sigma_{i}-a} h_{e}=\min _{e \in \sigma_{i}} h_{e}$ then the above is equivalent to

$$
t \leq \sum_{q=1}^{\min _{e} \in \sigma_{i}-a}\left(\prod_{e \in \sigma_{i}-a} \frac{h_{e}-q+1}{h_{e}}\right) \frac{q-1}{h_{a}}=\sum_{q=1}^{\min _{e \in \sigma_{i}} h_{e}}\left(\prod_{e \in \sigma_{i}-a} \frac{h_{e}-q+1}{h_{e}}\right) \frac{q-1}{h_{a}} .
$$

Similar inequalities can be derived for A- and S-moves as is demonstrated in Observation 4.1

Below we present the proofs of Lemmas 4.1- 4.5 and Theorem 5.1. We use the following notation. For any $X \subseteq M$ such that $h_{e}<n$ for all $e \in X$, we denote by $h^{X}$ the congestion vector with the congestion of each resource in $X$ being increased by 1 , while the congestion of all other resources remains unchanged. That is, $h_{e}^{X}=h_{e}+1$ for all $e \in X$ and $h_{e}^{X}=h_{e}$ for all $e \in M \backslash X$.

Proof of Lemma 4.1. Let $h$ be the congestion vector of $\sigma$. Let $i \in N$ be a player who can benefit from a unilateral deviation from $\sigma$. We have to show that there is a profitable A-, D- or S-move is available for $i$.

Clearly, if player $i$ deviates from strategy $\sigma_{i}$ to strategy $\sigma_{i}^{\prime}$ by applying a single A-, D- or S-move, then $\max \left\{\left|\sigma_{i} \backslash \sigma_{i}^{\prime}\right|, \quad\left|\sigma_{i}^{\prime} \backslash \sigma_{i}\right|\right\}=1$, and vice versa, if $\max \left\{\left|\sigma_{i} \backslash \sigma_{i}^{\prime}\right|,\left|\sigma_{i}^{\prime} \backslash \sigma_{i}\right|\right\}=1$ then $\sigma_{i}^{\prime}$ is obtained from $\sigma_{i}$ by applying exactly one such move. For simplicity of exposition, for any pair of sets $A$ and $B$, let $\mu(A, B)=\max \{|A \backslash B|,|B \backslash A|\}$.

Let $P D_{i}(\sigma)$ denote the set of all profitable deviations of $i$ from $\sigma$, that is

$$
P D_{i}(\sigma)=\left\{x_{i} \in \Sigma_{i}: C_{i}\left(\sigma_{-i}, x_{i}\right)<C_{i}(\sigma)\right\}
$$

and let $y_{i} \in \arg \min _{x_{i} \in P D_{i}(\sigma)} \mu\left(x_{i}, \sigma_{i}\right)$. We have to show that $\mu\left(y_{i}, \sigma_{i}\right)=1$, implying the existence of a profitable A-, D- or S-move.

Assume on the contrary that there is no profitable A-, D- or S-move. That is, $\mu\left(y_{i}, \sigma_{i}\right)>1$ (clearly, if $\mu\left(y_{i}, \sigma_{i}\right)<1$ then $\left.y_{i}=\sigma_{i}\right)$. Then, the following three inequalities hold for any $a \in \sigma_{i}$ and $b \notin \sigma_{i}$ :

$$
\begin{aligned}
& C_{i}(\sigma) \leq C_{i}\left(\sigma_{-i}, \sigma_{i}+b\right) \\
& C_{i}(\sigma) \leq C_{i}\left(\sigma_{-i}, \sigma_{i}-a\right) ; \\
& C_{i}(\sigma) \leq C_{i}\left(\sigma_{-i}, \sigma_{i}-a+b\right) ;
\end{aligned}
$$

Let $a \in \sigma_{i}$ and $b \notin \sigma_{i}$. By (4) and Observation 4.1, for any $a$ and $b$ as above we have

$$
h_{b}+1 \geq h_{a} .
$$

By (2) and Observation 4.1, for any $b \notin \sigma_{i}$ we get

$$
t \geq \sum_{q=1}^{\min _{\sigma_{i}} h_{e}}\left(\prod_{e \in \sigma_{i}} \frac{h_{e}-q+1}{h_{e}}\right) \frac{q-1}{h_{b}+1} .
$$

By (3), for any $a \in \sigma_{i}$,

$$
\begin{gathered}
\sum_{q=1}^{\min _{\sigma_{i}} h_{e}} \prod_{e \in \sigma_{i}} \frac{h_{e}-q+1}{h_{e}}+\left|\sigma_{i}\right| t \leq \sum_{q=1}^{\min _{\sigma_{i}-a} h_{e}} \prod_{e \in \sigma_{i}-a} \frac{h_{e}-q+1}{h_{e}}+\left(\left|\sigma_{i}\right|-1\right) t \\
\Rightarrow t \leq \sum_{q=1}^{\min _{\sigma_{i}-a} h_{e}} \prod_{e \in \sigma_{i}-a} \frac{h_{e}-q+1}{h_{e}}-\sum_{q=1}^{\min _{\sigma_{i}}} \prod_{e \in \sigma_{i}} \frac{h_{e}-q+1}{h_{e}} .
\end{gathered}
$$

We consider separately each of the following three cases: (i) $\left|\sigma_{i} \backslash y_{i}\right|=0$, (ii) $\left|y_{i} \backslash \sigma_{i}\right|=0$, and (iii) both $\left|y_{i} \backslash \sigma_{i}\right|$ and $\left|\sigma_{i} \backslash y_{i}\right|$ are positive. 
(i) $\left|\boldsymbol{\sigma}_{\boldsymbol{i}} \backslash \boldsymbol{y}_{\boldsymbol{i}}\right|=\mathbf{0}$ :

If $\left|\sigma_{i} \backslash y_{i}\right|=0$ (i.e., $\sigma_{i} \subsetneq y_{i}$ ), then let $\hat{b} \in \arg \max _{e \in y_{i} \backslash \sigma_{i}} h_{e}^{y_{i} \backslash \sigma_{i}}$, and consider the strategy profile $y_{i}^{\prime}=y_{i}-\hat{b}$ which is obtained by a D-move of $i$ from $\left(\sigma_{-i}, y_{i}\right)$. Clearly,

$$
\mu\left(y_{i}^{\prime}, \sigma_{i}\right)=\left|y_{i}^{\prime} \backslash \sigma_{i}\right|<\left|y_{i} \backslash \sigma_{i}\right|=\mu\left(y_{i}, \sigma_{i}\right)
$$

We show below that $y_{i}^{\prime}$ is a profitable deviation of $i$ from $\sigma$, and thus contradicts the choice of $y_{i}$. Specifically, we demonstrate that $C_{i}\left(\sigma_{-i}, y_{i}^{\prime}\right) \leq C_{i}\left(\sigma_{-i}, y_{i}\right)$, which implies that $y_{i}^{\prime} \in P D_{i}(\sigma)$.

Assume on the contrary that $C_{i}\left(\sigma_{-i}, y_{i}^{\prime}\right)>C_{i}\left(\sigma_{-i}, y_{i}\right)$. By $\sqrt{5}$ and the choice of $\hat{b}$, Observation 4.1 implies that

$$
\begin{aligned}
t & <\sum_{q=1}^{\min _{y_{i}} h_{e}^{y_{i} \backslash \sigma_{i}}}\left(\prod_{e \in y_{i}-\hat{b}} \frac{h_{e}^{y_{i} \backslash \sigma_{i}}-q+1}{h_{e}^{y_{i} \backslash \sigma_{i}}}\right) \frac{q-1}{h_{\hat{b}}+1} \\
& =\sum_{q=1}^{\min _{y_{i}} h_{e}^{y_{i} \backslash \sigma_{i}}}\left(\prod_{e \in y_{i} \cap \sigma_{i}} \frac{h_{e}-q+1}{h_{e}} \prod_{e \in\left(y_{i} \backslash \sigma_{i}\right)-\hat{b}} \frac{h_{e}^{y_{i} \backslash \sigma_{i}}-q+1}{h_{e}^{y_{i} \backslash \sigma_{i}}}\right) \frac{q-1}{h_{\hat{b}}+1} .
\end{aligned}
$$

Since $\sigma_{i} \subsetneq y_{i}$ and $\left|y_{i} \backslash \sigma_{i}\right|>1$ then $y_{i} \cap \sigma_{i}=\sigma_{i}, \min _{y_{i}} h_{e}^{y_{i} \backslash \sigma_{i}} \leq \min _{\sigma_{i}} h_{e}$ and $\prod_{e \in\left(y_{i} \backslash \sigma_{i}\right)-\hat{b}} \frac{h_{e}^{y_{i} \backslash \sigma_{i}}-q+1}{h_{e}^{y_{i} \backslash \sigma_{i}}}<1$ for any $1<q \leq \min _{y_{i}} h_{e}^{y_{i} \backslash \sigma_{i}}$. This, coupled with 8 , yields

$$
t<\sum_{q=1}^{\min _{\sigma_{i}} h_{e}}\left(\prod_{e \in \sigma_{i}} \frac{h_{e}-q+1}{h_{e}}\right) \frac{q-1}{h_{\hat{b}}+1}
$$

which contradicts 6 .

(ii) $\left|\boldsymbol{y}_{\boldsymbol{i}} \backslash \boldsymbol{\sigma}_{\boldsymbol{i}}\right|=\mathbf{0}$ :

If $\left|y_{i} \backslash \sigma_{i}\right|=0$ (i.e., $y_{i} \subsetneq \sigma_{i}$ ), then let $\hat{a} \in \arg \max _{e \in \sigma_{i} \backslash y_{i}} h_{e}$, and consider the strategy profile $y_{i}^{\prime}=y_{i}+\hat{a}$ which is obtained by an A-move of $i$ from $\left(\sigma_{-i}, y_{i}\right)$. Clearly,

$$
\mu\left(y_{i}^{\prime}, \sigma_{i}\right)=\left|\sigma_{i} \backslash y_{i}^{\prime}\right|<\left|\sigma_{i} \backslash y_{i}\right|=\mu\left(y_{i}, \sigma_{i}\right) .
$$

As before, we show that $y_{i}^{\prime}$ is a profitable deviation of $i$ from $\sigma$, and thus contradicts the choice of $y_{i}$. Specifically, we demonstrate that $C_{i}\left(\sigma_{-i}, y_{i}^{\prime}\right) \leq C_{i}\left(\sigma_{-i}, y_{i}\right)$, which implies that $y_{i}^{\prime} \in P D_{i}(\sigma)$. Assume on the contrary that $C_{i}\left(\sigma_{-i}, y_{i}^{\prime}\right)>C_{i}\left(\sigma_{-i}, y_{i}\right)$. Then, by Observation 4.1

$$
t>\sum_{q=1}^{\min _{y_{i}} h_{e}}\left(\prod_{e \in y_{i}} \frac{h_{e}-q+1}{h_{e}}\right) \frac{q-1}{h_{\hat{a}}} .
$$

By the choice of $\hat{a}$ and since $\left|\sigma_{i} \backslash y_{i}\right|>1$ we conclude that there exists $e \in \sigma_{i} \backslash y_{i} \subseteq \sigma_{i}$ such that $h_{e} \leq h_{\hat{a}}$, which implies that $\min _{e \in \sigma_{i}-\hat{a}}=\min _{\sigma_{i}}$. Then, (7) implies that

$$
t \leq \sum_{q=1}^{\min _{\sigma_{i}} h_{e}}\left(\prod_{e \in \sigma_{i}-\hat{a}} \frac{h_{e}-q+1}{h_{e}}\right) \frac{q-1}{h_{\hat{a}}} .
$$

Since $y_{i} \subsetneq \sigma_{i}-\hat{a}$ then $\min _{\sigma_{i}} h_{e} \leq \min _{y_{i}} h_{e}$ and $\prod_{e \in \sigma_{i}-\hat{a}} \frac{h_{e}-q+1}{h_{e}}<\prod_{e \in y_{i}} \frac{h_{e}-q+1}{h_{e}}$ for all $1 \leq q \leq$ $\min _{\sigma_{i}} h_{e}$, which implies that $(10)$ contradicts $(9)$.

(iii) $\left|y_{i} \backslash \sigma_{i}\right|>0$ and $\left|\sigma_{i} \backslash y_{i}\right|>0$ :

If both $\left|y_{i} \backslash \sigma_{i}\right|$ and $\left|\sigma_{i} \backslash y_{i}\right|$ are positive, then let $a \in \sigma_{i} \backslash y_{i}$ and $b \in y_{i} \backslash \sigma_{i}$, and consider a strategy profile $y_{i}^{\prime}=\left(y_{i}-b+a\right)$ which is obtained by an S-move from $\left(\sigma_{-i}, y_{i}\right)$. By (5) and Observation 4.1 we get $C_{i}\left(\sigma_{-i}, y_{i}^{\prime}\right) \leq C_{i}\left(\sigma_{-i}, y_{i}\right)$, implying that $y_{i}^{\prime} \in P D_{i}(\sigma)$. Clearly, $\left|y_{i}^{\prime} \backslash \sigma_{i}\right|<\left|y_{i} \backslash \sigma_{i}\right|$ and $\left|\sigma_{i} \backslash y_{i}^{\prime}\right|<\left|\sigma_{i} \backslash y_{i}\right|$. Therefore, $\mu\left(y_{i}^{\prime}, \sigma_{i}\right)<\mu\left(y_{i}, \sigma_{i}\right)$, which contradicts the choice of $y_{i}$. This completes the proof.

Proof of Lemma 4.2. The lemma follows directly from Observation 4.1 and the discussion below. 
$\Rightarrow$ Let $\sigma$ be an S-stable strategy profile with its corresponding congestion vector $h$. Assume on the contrary that there are $a, b \in M$ such that $h_{b}+1<h_{a}$. Then, there exists $i \in N$ such that $a \in \sigma_{i}$ and $b \in M \backslash \sigma_{i}$, and

$$
\begin{aligned}
& C_{i}\left(\sigma_{-i}, \sigma_{i}-a+b\right)=\sum_{q=1}^{\min _{\sigma_{i}-a+b} h_{e}^{b}} \prod_{e \in \sigma_{i}-a+b} \frac{h_{e}^{b}-q+1}{h_{e}^{b}}+\left|\sigma_{i}\right| t \\
& <\sum_{q=1}^{\min _{\sigma_{i}} h_{e}} \prod_{e \in \sigma_{i}} \frac{h_{e}-q+1}{h_{e}}+\left|\sigma_{i}\right| t=C_{i}(\sigma),
\end{aligned}
$$

in contradiction to the S-stability of $\sigma$.

$\Leftarrow$ Let $\sigma$ be a profile with a nearly even congestion on the resources, and let $h$ be its corresponding congestion vector. Assume on the contrary that there exist $i \in N$ and $a \in \sigma_{i}, b \in M \backslash \sigma_{i}$, such that $C_{i}\left(\sigma_{-i}, \sigma_{i}-a+b\right)<C_{i}(\sigma)$. Then,

$$
\sum_{q=1}^{\min _{\sigma_{i}-a+b} h_{e}^{b}} \prod_{e \in \sigma_{i}-a+b} \frac{h_{e}^{b}-q+1}{h_{e}^{b}}+\left|\sigma_{i}\right| t<\sum_{q=1}^{\min _{\sigma_{i}} h_{e}} \prod_{e \in \sigma_{i}} \frac{h_{e}-q+1}{h_{e}}+\left|\sigma_{i}\right| t .
$$

Since $\sigma$ is nearly-even then $h_{b}+1 \geq h_{a}$, implying that $\min _{\sigma_{i}-a+b} h_{e}^{b} \geq \min _{\sigma_{i}} h_{e}$. Therefore, there exists $1 \leq q \leq \min _{\sigma_{i}} h_{e}$ such that $\prod_{e \in \sigma_{i}-a+b} \frac{h_{e}^{b}-q+1}{h_{e}^{b}}<\prod_{e \in \sigma_{i}} \frac{h_{e}-q+1}{h_{e}}$, implying $h_{b}+1<h_{a}$, a contradiction.

Proof of Lemma 4.3. We prove the lemma for the one- and the two-step addition, separately.

(i) One-step addition:

Suppose $\sigma^{\prime}$ is obtained from $\sigma$ by a one-step addition of $a$ by player $i$, that is, $\sigma^{\prime}=\left(\sigma_{-i}, \sigma_{i}+a\right)$. Then, $C_{i}\left(\sigma^{\prime}\right)<C_{i}(\sigma) \Rightarrow C_{i}\left(\sigma^{\prime}\right)<C_{i}\left(\sigma_{-i}^{\prime}, \sigma_{i}^{\prime}-a\right)$, i.e. dropping resource $a$ is not a profitable policy for $i$. Since $h_{a}\left(\sigma^{\prime}\right)=h_{a}(\sigma)+1 \geq h_{b}(\sigma)=h_{b}\left(\sigma^{\prime}\right)$ for any $b \in \sigma_{i}=\sigma_{i}^{\prime}-a$ then $\min _{e \in \sigma_{i}^{\prime}-a} \leq$ $\min _{e \in \sigma_{i}^{\prime}-b}$ and $\frac{h_{b}\left(\sigma^{\prime}\right)-q+1}{h_{b}\left(\sigma^{\prime}\right)} \leq \frac{h_{a}\left(\sigma^{\prime}\right)-q+1}{h_{a}\left(\sigma^{\prime}\right)}$ for any $1 \leq q \leq \min _{e \in \sigma_{i}^{\prime}-a}$. Therefore,

$$
\begin{aligned}
C_{i}\left(\sigma_{-i}^{\prime}, \sigma_{i}^{\prime}-a\right) & =\sum_{q=1}^{\min _{e \in \sigma_{i}^{\prime}-a} h_{e}\left(\sigma^{\prime}\right)} \prod_{e \in \sigma_{i}^{\prime}-a} \frac{h_{e}\left(\sigma^{\prime}\right)-q+1}{h_{e}\left(\sigma^{\prime}\right)}+\left(\left|\sigma_{i}^{\prime}\right|-1\right) t \\
\leq & \sum_{q=1}^{\min _{e \in \sigma_{i}^{\prime}-b} h_{e}\left(\sigma^{\prime}\right)} \prod_{e \in \sigma_{i}^{\prime}-b} \frac{h_{e}\left(\sigma^{\prime}\right)-q+1}{h_{e}\left(\sigma^{\prime}\right)}+\left(\left|\sigma_{i}^{\prime}\right|-1\right) t=C_{i}\left(\sigma_{-i}^{\prime}, \sigma_{i}^{\prime}-b\right),
\end{aligned}
$$

implying $C_{i}\left(\sigma^{\prime}\right)<C_{i}\left(\sigma_{-i}^{\prime}, \sigma_{i}^{\prime}-b\right)$. That is, no profitable D-move is available for player $i$.

Let $k \neq i$. Then, $\sigma_{k}^{\prime}=\sigma_{k}$. We have to show that dropping any resource $b \neq a$ is not profitable for player $k$. Assuming on the contrary that $C_{k}\left(\sigma_{-k}^{\prime}, \sigma_{k}^{\prime}-b\right)<C_{k}\left(\sigma^{\prime}\right)$, by Observation 4.1 we get

$$
t>\sum_{q=1}^{\min _{e \in \sigma_{k}^{\prime}} h_{e}\left(\sigma^{\prime}\right)}\left(\prod_{e \in \sigma_{k}^{\prime}-b} \frac{h_{e}\left(\sigma^{\prime}\right)-q+1}{h_{e}\left(\sigma^{\prime}\right)}\right) \frac{q-1}{h_{b}\left(\sigma^{\prime}\right)}
$$

Let $\hat{b} \in \arg \max _{e \in \sigma_{k}-a} h_{e}(\sigma)$. By the D-stability of $\sigma, C_{k}(\sigma) \leq C_{k}\left(\sigma_{-k}, \sigma_{k}-\hat{b}\right)$, implying

$$
\begin{aligned}
& \sum_{q=1}^{\min _{e \in \sigma_{k}}} \prod_{e \in \sigma_{k}} \frac{h_{e}(\sigma)-q+1}{h_{e}(\sigma)}+\left|\sigma_{k}\right| t \\
& \leq \sum_{q=1}^{\min _{e \in \sigma_{k}-\hat{b}} h_{e}(\sigma)} \prod_{e \in \sigma_{k}-\hat{b}} \frac{h_{e}(\sigma)-q+1}{h_{e}(\sigma)}+\left(\left|\sigma_{k}\right|-1\right) t .
\end{aligned}
$$


Since $a$ is $\sigma$-light then $h_{\hat{b}}(\sigma) \geq h_{a}(\sigma)$, implying $\hat{b} \in \arg \max _{e \in \sigma_{k}} h_{e}(\sigma)$. Then, $\min _{e \in \sigma_{k}-\hat{b}} h_{e}(\sigma)=$ $\min _{e \in \sigma_{k}} h_{e}(\sigma)$. Therefore, by Observation 4.1 the above yields

$$
t \leq \sum_{q=1}^{\min _{e \in \sigma_{k}} h_{e}(\sigma)}\left(\prod_{e \in \sigma_{k}-\hat{b}} \frac{h_{e}(\sigma)-q+1}{h_{e}(\sigma)}\right) \frac{q-1}{h_{\hat{b}}(\sigma)} .
$$

By 12 and 13 ,

$$
\begin{aligned}
& \sum_{q=1}^{\min _{e \in \sigma_{k}^{\prime}} h_{e}\left(\sigma^{\prime}\right)}\left(\prod_{e \in \sigma_{k}^{\prime}-\hat{b}} \frac{h_{e}\left(\sigma^{\prime}\right)-q+1}{h_{e}\left(\sigma^{\prime}\right)}\right) \frac{q-1}{h_{\hat{b}}\left(\sigma^{\prime}\right)} \\
< & \sum_{q=1}^{\min _{e \in \sigma_{k}} h_{e}(\sigma)}\left(\prod_{e \in \sigma_{k}-\hat{b}} \frac{h_{e}(\sigma)-q+1}{h_{e}(\sigma)}\right) \frac{q-1}{h_{\hat{b}}(\sigma)} .
\end{aligned}
$$

Since $\sigma_{k}^{\prime}=\sigma_{k}$ and $h_{e}\left(\sigma^{\prime}\right) \geq h_{e}(\sigma)$ for all $e \in M$ then $\min _{e \in \sigma_{k}^{\prime}} h_{e}\left(\sigma^{\prime}\right) \geq \min _{e \in \sigma_{k}} h_{e}(\sigma)$, implying that

$$
\left(\prod_{e \in \sigma_{k}^{\prime}-\hat{b}} \frac{h_{e}\left(\sigma^{\prime}\right)-q+1}{h_{e}\left(\sigma^{\prime}\right)}\right) \frac{q-1}{h_{\hat{b}}\left(\sigma^{\prime}\right)}<\left(\prod_{e \in \sigma_{k}-\hat{b}} \frac{h_{e}(\sigma)-q+1}{h_{e}(\sigma)}\right) \frac{q-1}{h_{\hat{b}}(\sigma)}
$$

for some $1 \leq q \leq \min _{e \in \sigma_{k}} h_{e}(\sigma)$. The above yields $h_{e}\left(\sigma^{\prime}\right)<h_{e}(\sigma)$ for some $e \in \sigma_{k}-\hat{b}$, a contradiction. Hence, dropping resource $\hat{b}$ is not a profitable policy for $k$. Since $\hat{b} \in \arg \max _{e \in \sigma_{k}-a} h_{e}(\sigma)$, $\sigma_{k}^{\prime}=\sigma_{k}$ and $h_{e}\left(\sigma^{\prime}\right)=h_{e}(\sigma)$ for all $e \neq a$ then $\hat{b} \in \arg \max _{e \in \sigma_{k}^{\prime}-a} h_{e}\left(\sigma^{\prime}\right)$. As before, this yields $C_{k}\left(\sigma_{-k}^{\prime}, \sigma_{k}^{\prime}-\hat{b}\right) \leq C_{k}\left(\sigma_{-k}^{\prime}, \sigma_{k}^{\prime}-b\right)$ for any $b \in \sigma_{k}^{\prime}-a$, implying $C_{k}\left(\sigma^{\prime}\right) \leq C_{k}\left(\sigma_{-k}^{\prime}, \sigma_{k}^{\prime}-b\right)$. Hence, the only possible profitable D-move for player $k$ is with the added resource $a$.

\section{ii. Two-step addition:}

Now suppose that $\sigma^{\prime}$ is obtained by a two-step addition of $a$. More precisely, let $\sigma^{\prime}=$ $\left(\sigma_{-\{i, j\}}, \sigma_{i}+a^{\prime}, \sigma_{j}-a^{\prime}+a\right)$, where $a^{\prime}$ is $\sigma$-heavy and $a$ is $\sigma$-light. Then, $C_{i}\left(\sigma_{-i}, \sigma_{i}+a^{\prime}\right)<C_{i}(\sigma)$ yields

$$
\begin{gathered}
t<\sum_{q=1}^{\min _{e \in \sigma_{i}} h_{e}(\sigma)} \prod_{e \in \sigma_{i}} \frac{h_{e}(\sigma)-q+1}{h_{e}(\sigma)}- \\
\sum_{q=1}^{\min _{e \in \sigma_{i}+a^{\prime}} h_{e}^{\left(\sigma-i, \sigma_{i}+a^{\prime}\right)}}\left(\prod_{e \in \sigma_{i}} \frac{h_{e}(\sigma)-q+1}{h_{e}(\sigma)}\right) \frac{h_{a^{\prime}}(\sigma)-q+2}{h_{a^{\prime}}(\sigma)+1} .
\end{gathered}
$$

Since $h_{a^{\prime}}^{\left(\sigma_{-i}, \sigma_{i}+a^{\prime}\right)}=h_{a^{\prime}}(\sigma)+1>h_{e}(\sigma)=h_{e}^{\left(\sigma_{-i}, \sigma_{i}+a^{\prime}\right)}$ for all $e \in M-a^{\prime}$, by Observation 4.1 the above yields

$$
t<\sum_{q=1}^{\min _{e \in \sigma_{i}} h_{e}(\sigma)}\left(\prod_{e \in \sigma_{i}} \frac{h_{e}(\sigma)-q+1}{h_{e}(\sigma)}\right) \frac{q-1}{h_{a^{\prime}}(\sigma)+1} .
$$

Since $a^{\prime}$ is $\sigma$-heavy and $a$ is $\sigma$-light, then $h_{a^{\prime}}\left(\sigma^{\prime}\right)=h_{a^{\prime}}(\sigma) \geq h_{e}\left(\sigma^{\prime}\right) \geq h_{e}(\sigma)$, for all $e \in M$. Then, by (14), we get

$$
t<\sum_{q=1}^{\min _{e \in \sigma_{i}^{\prime}} h_{e}\left(\sigma^{\prime}\right)}\left(\prod_{e \in \sigma_{i}^{\prime}-a^{\prime}} \frac{h_{e}\left(\sigma^{\prime}\right)-q+1}{h_{e}\left(\sigma^{\prime}\right)}\right) \frac{q-1}{h_{a^{\prime}}\left(\sigma^{\prime}\right)},
$$

implying $C_{i}\left(\sigma^{\prime}\right)<C_{i}\left(\sigma_{-i}^{\prime}, \sigma_{i}^{\prime}-a^{\prime}\right)$, i.e. dropping resource $a$ is not a profitable policy for $i$. Since $h_{a^{\prime}}\left(\sigma^{\prime}\right) \geq h_{e}\left(\sigma^{\prime}\right)$ for all $e \in M$ then the above yields $C_{i}\left(\sigma^{\prime}\right)<C_{i}\left(\sigma_{-i}^{\prime}, \sigma_{i}^{\prime}-e\right)$ for any $e \in \sigma_{i}^{\prime}$, implying that no profitable D-move is available for player $i$.

Consider now player $j$. We have to show that no profitable D-move is available for $j$. We demonstrate below that the required follows directly from the D-stability of $\sigma$. That is, $C_{j}(\sigma) \leq$ $C_{j}\left(\sigma_{-j}, \sigma_{j}-e\right)$ for all $e \in \sigma_{j}$, implies $C_{j}\left(\sigma^{\prime}\right) \leq C_{j}\left(\sigma_{-j}^{\prime}, \sigma_{j}^{\prime}-e^{\prime}\right)$ for all $e^{\prime} \in \sigma_{j}^{\prime}$. Since $a^{\prime}$ is $\sigma$-heavy, 
$a$ is $\sigma$-light and $\sigma$ is nearly-even, then $h_{a}\left(\sigma^{\prime}\right)=h_{a}(\sigma)+1=h_{a^{\prime}}(\sigma)$. Recall that $\sigma_{j}^{\prime}=\sigma_{j}-a^{\prime}+a$ and $h_{e}\left(\sigma^{\prime}\right)=h_{e}(\sigma)$ for all $e \in M-a$. Then, for any $e \in \sigma_{j}, e^{\prime} \in \sigma_{j}^{\prime}$, we have

$$
C_{j}\left(\sigma^{\prime}\right)=C_{j}(\sigma) \leq C_{j}\left(\sigma_{-j}, \sigma_{j}-e\right)=C_{j}\left(\sigma_{-j}^{\prime}, \sigma_{j}^{\prime}-e^{\prime}\right),
$$

as required.

For any player $k \neq i, j$, the proof we provided in the first case, is valid as well.

Proof of Lemma 4.4. If $\Sigma^{2}$ contains a pure strategy Nash equilibrium strategy profile, we are done. Otherwise, let us first show that $\Sigma^{2}$ is not empty. By the definition of $\Sigma^{2}$, it suffices to show that $\Sigma^{1}-$ the set of even D-stable profiles - is not empty. Recall that $m<n$ and consider the D-stable strategy profile $\sigma=\left(e_{i \bmod m+1}\right)_{i \in N}$. If $m$ divides $n$ then $\sigma$ is $\frac{n}{m}$-even, and thus $\Sigma^{1}$ is not empty. Otherwise, $m$ does not divide $n$, and $\sigma$ is nearly-even, but not even. Observe that in $\sigma$, each player uses exactly one resource, and $n \bmod m$ resources are chosen by $\left\lceil\frac{n}{m}\right\rceil=\left\lfloor\frac{n}{m}\right\rfloor+1$ players, while the other $m-(n \bmod m)$ resources are selected by $\left\lfloor\frac{n}{m}\right\rfloor$ players. We modify $\sigma$ to a $\left\lceil\frac{n}{m}\right\rceil$-even strategy profile in the following way. We divide the $n$ players into two groups. The first group $\{1, \ldots, m-(n \bmod m)\}$ contains $m-(n \bmod m)$ players, and the remaining $n-(m-(n \bmod m))$ players form the second group $\{m-(n \bmod m)+1, \ldots, n\}$. Player $i$ in the first group assigns his task to two resources, $e_{i \bmod m+1}$ and $e_{(i+n) \bmod m+1}$; player $i$ in the second group assigns his task to a single resource $e_{i \bmod m+1}$. We denote the modified profile by $\sigma^{\prime}$. We show below that $\sigma^{\prime}$ is D-stable, implying that $\Sigma^{1}$ is not empty.

By our assumption, the previously considered profile $\sigma=\left(e_{i} \bmod m+1\right)_{i \in N}$ is not A-stable, i.e. there exists a player $i \in N$ such that $C_{i}(\sigma)>C_{i}\left(\sigma_{-i}, \sigma_{i}+a\right)$ for some $a \in M \backslash \sigma_{i}$. We claim that there exist $i \in N$ and $a \in M$, as above, such that $a$ is $\sigma$-light. If $n \bmod m<m-1$ then our claim follows immediately. Otherwise, there is only one $\sigma$-light resource, $e_{1}$. If no player in $N$ wishes to add $e_{1}$ to his strategy then player $i$, who wishes to apply an A-move with some $\sigma$-heavy resource $a$, currently uses the resource $e_{1}$. In this case $C_{i}(\sigma)>C_{i}\left(\sigma_{-i}, \sigma_{i}+a\right)=C_{i}\left(\sigma_{-i}, e_{1}+a\right)$, which yields

$$
\begin{gathered}
\sum_{q=1}^{\left\lfloor\frac{n}{m}\right\rfloor} \frac{\left\lfloor\frac{n}{m}\right\rfloor-q+1}{\left\lfloor\frac{n}{m}\right\rfloor}+t>\sum_{q=1}^{\left\lfloor\frac{n}{m}\right\rfloor} \frac{\left\lfloor\frac{n}{m}\right\rfloor-q+1}{\left\lfloor\frac{n}{m}\right\rfloor} \cdot \frac{\left\lceil\frac{n}{m}\right\rceil-q+2}{\left\lceil\frac{n}{m}\right\rceil+1}+2 t \\
\Rightarrow t<\sum_{q=1}^{\left\lfloor\frac{n}{m}\right\rfloor} \frac{\left\lfloor\frac{n}{m}\right\rfloor-q+1}{\left\lfloor\frac{n}{m}\right\rfloor} \cdot \frac{q-1}{\left\lceil\frac{n}{m}\right\rceil+1}<\sum_{q=1}^{\left\lceil\frac{n}{m}\right\rceil} \frac{\left\lceil\frac{n}{m}\right\rceil-q+1}{\left\lceil\frac{n}{m}\right\rceil} \cdot \frac{q-1}{\left\lceil\frac{n}{m}\right\rceil} \\
\Rightarrow \sum_{q=1}^{\left\lceil\frac{n}{m}\right\rceil} \frac{\left\lceil\frac{n}{m}\right\rceil-q+1}{\left\lceil\frac{n}{m}\right\rceil}+t>\sum_{q=1}^{\left\lceil\frac{n}{m}\right\rceil}\left(\frac{\left\lceil\frac{n}{m}\right\rceil-q+1}{\left\lceil\frac{n}{m}\right\rceil}\right)^{2}+2 t,
\end{gathered}
$$

implying that an A-move with the $\sigma$-light resource $e_{1}$ is profitable for any player currently using some $\sigma$-heavy resource. Thus, there are player $i \in N$ and a $\sigma$-light resource $a \notin \sigma_{i}$ such that an A-move with $a$ is profitable for $i$.

We turn now to show the D-stability of $\sigma^{\prime}$. We do so by observing that if player $i$ uses in $\sigma$ a heavy resource with the congestion of $\left\lceil\frac{n}{m}\right\rceil$, and it is profitable for him to use two such resources instead of one (note that if player $i$ joins a $\sigma$-light resource $a$, the congestion of $a$ increases by 1 , from $\left\lfloor\frac{n}{m}\right\rfloor$ to $\left.\left\lceil\frac{n}{m}\right\rceil\right)$, then there is no profitable D-move from the modified profile, $\sigma^{\prime}$, for any player $j \in N$. Namely, if $C_{i}(\sigma)>C_{i}\left(\sigma_{-i}, \sigma_{i}+a\right)$, where $a$ is a $\sigma$-light resource and $\sigma_{i}$ consists of a single $\sigma$-heavy resource, then $C_{j}\left(\sigma^{\prime}\right)<C_{j}\left(\sigma_{-j}^{\prime}, \sigma_{j}^{\prime}-b\right)$ for any $j \in N$ with $\left|\sigma_{j}^{\prime}\right|>1$ and $b \in \sigma_{j}^{\prime}$. Otherwise, if player $i$ uses in $\sigma$ a light resource with the congestion of $\left\lfloor\frac{n}{m}\right\rfloor$, and it is beneficial for him to add a $\sigma$-light resource $a$, then using two resources, one with the congestion of $\left\lfloor\frac{n}{m}\right\rfloor$ and one with the congestion of $\left\lceil\frac{n}{m}\right\rceil$, is better for $i$ than using only a single $\sigma$-light resource. This in turn yields that

$$
\begin{aligned}
& \sum_{q=1}^{\left\lfloor\frac{n}{m}\right\rfloor} \frac{\left\lfloor\frac{n}{m}\right\rfloor-q+1}{\left\lfloor\frac{n}{m}\right\rfloor}+t>\sum_{q=1}^{\left\lfloor\frac{n}{m}\right\rfloor} \frac{\left\lfloor\frac{n}{m}\right\rfloor-q+1}{\left\lfloor\frac{n}{m}\right\rfloor} \cdot \frac{\left\lfloor\frac{n}{m}\right\rfloor-q+2}{\left\lfloor\frac{n}{m}\right\rfloor+1}+2 t \\
& \Rightarrow t<\sum_{q=1}^{\left\lfloor\frac{n}{m}\right\rfloor} \frac{\left\lfloor\frac{n}{m}\right\rfloor-q+1}{\left\lfloor\frac{n}{m}\right\rfloor} \cdot \frac{q-1}{\left\lfloor\frac{n}{m}\right\rfloor+1}<\sum_{q=1}^{\left\lceil\frac{n}{m}\right\rceil} \frac{\left\lceil\frac{n}{m}\right\rceil-q+1}{\left\lceil\frac{n}{m}\right\rceil} \cdot \frac{q-1}{\left\lceil\frac{n}{m}\right\rceil}
\end{aligned}
$$




$$
\Rightarrow \sum_{q=1}^{\left\lceil\frac{n}{m}\right\rceil}\left(\frac{\left\lceil\frac{n}{m}\right\rceil-q+1}{\left\lceil\frac{n}{m}\right\rceil}\right)^{2}+2 t<\sum_{q=1}^{\left\lceil\frac{n}{m}\right\rceil} \frac{\left\lceil\frac{n}{m}\right\rceil-q+1}{\left\lceil\frac{n}{m}\right\rceil}+t .
$$

Hence, $C_{j}\left(\sigma^{\prime}\right)<C_{i}\left(\sigma_{-j}^{\prime}, \sigma_{j}^{\prime}-b\right)$ for any $j \in N$ with $\left|\sigma_{j}^{\prime}\right|>1$ and $b \in \sigma_{j}^{\prime}$. Therefore, $\sigma^{\prime}$ is D-stable. Thus, we have shown that $\Sigma^{1}$, the set of even D-stable strategy profiles, is not empty. Therefore, $\Sigma^{2}$ is not empty as well.

Let $C_{N}(\sigma)=\sum_{j \in N} C_{j}(\sigma)$ denotes the group cost of the players, and let $\Sigma^{3} \subseteq \Sigma^{2}$ be the non-empty subset of all profiles in $\Sigma^{2}$ of minimum group cost. That is,

$$
\Sigma^{3}=\arg \min _{\sigma \in \Sigma^{2}} \sum_{j \in N} C_{j}(\sigma)=\arg \min _{\sigma \in \Sigma^{2}} C_{N}(\sigma) .
$$

We show below that $\Sigma^{3}$ contains a post-addition D-stable strategy profile. That is, by Definition 4.4 , it should be shown that there is $\sigma \in \Sigma^{3}$ such that for all $j \in N$ with $\left|\sigma_{j}\right|>1$,

$$
t \leq \sum_{q=1}^{H^{\sigma}}\left(\frac{H^{\sigma}-q+1}{H^{\sigma}}\right)^{\left|\sigma_{j}\right|-1} \cdot \frac{q-1}{H^{\sigma}+1} .
$$

Let $\sigma \in \Sigma^{3}$ and let $M(\sigma)$ be the subset of all resources for which there exists a profitable (one-step) addition by any of the players. First, we show that (15) holds for all $j \in N$ with $\left|\sigma_{j}\right|>1$ such that $\sigma_{j} \cap M(\sigma) \neq \varnothing$. That is, 15 is true for all those players with one of their resources being desired by another player.

Let $a \in M(\sigma)$, and let $\sigma^{\prime}$ be the strategy profile that is obtained from $\sigma$ by the (one-step) addition of $a$ by player $i$. Assume on the contrary that there is a player $j$ with $a \in \sigma_{j},\left|\sigma_{j}\right|>1$, who would like to remove $a$ from the set of resources he uses. This implies that

$$
t>\sum_{q=1}^{H^{\sigma}}\left(\frac{H^{\sigma}-q+1}{H^{\sigma}}\right)^{\left|\sigma_{j}\right|-1} \cdot \frac{q-1}{H^{\sigma}+1} .
$$

Let $\sigma^{\prime \prime}=\left(\sigma_{-j}^{\prime}, \sigma_{j}^{\prime}-a\right)$. Below we demonstrate that $\sigma^{\prime \prime}$ is a D-stable strategy profile and, since $\sigma^{\prime \prime}$ and $\sigma$ correspond to the same congestion vector, we conclude that $\sigma^{\prime \prime}$ lies in $\Sigma^{2}$. In addition, we show that $C_{N}\left(\sigma^{\prime \prime}\right)<C_{N}(\sigma)$, contradicting the fact that $\sigma \in \Sigma^{3}$.

To show that $\sigma^{\prime \prime} \in \Sigma^{0}$ we note that since $H^{\sigma^{\prime \prime}}=H^{\sigma}$ and $\sigma \in \Sigma^{0}$, there are no profitable D-moves for any player $k \neq i, j$. It remains to show that there are no profitable D-moves for players $i$ and $j$ as well. Since $C_{i}\left(\sigma^{\prime}\right)<C_{i}(\sigma), h_{a}\left(\sigma^{\prime \prime}\right)<h_{a}\left(\sigma^{\prime}\right)$ and $h_{e}\left(\sigma^{\prime \prime}\right)=h_{e}\left(\sigma^{\prime}\right)$ for all $e \in M-a$ then $C_{i}\left(\sigma^{\prime \prime}\right) \leq C_{i}\left(\sigma^{\prime}\right)<C_{i}(\sigma)$. Now, since $h_{e}\left(\sigma^{\prime \prime}\right)=h_{e}(\sigma)$ for all $e \in M$ and $\sigma^{\prime \prime}$ is even, we get $C_{i}\left(\sigma^{\prime \prime}\right)<C_{i}(\sigma)=C_{i}\left(\sigma_{-i}^{\prime \prime}, \sigma_{i}^{\prime \prime}-a\right)=$ $C_{i}\left(\sigma_{-i}^{\prime \prime}, \sigma_{i}^{\prime \prime}-e\right)$ for all $e \in \sigma_{i}^{\prime \prime}$, implying that no profitable D-move is available to player $i$. By the D-stability of $\sigma$, for player $j$ and for all $e \in \sigma_{j}, C_{j}(\sigma) \leq C_{j}\left(\sigma_{-j}, \sigma_{j}-e\right)$. Hence, by Observation 4.1 .

$$
\begin{aligned}
t & \leq \sum_{q=1}^{H^{\sigma}}\left(\frac{H^{\sigma}-q+1}{H^{\sigma}}\right)^{\left|\sigma_{j}\right|-1} \cdot \frac{q-1}{H^{\sigma}} \\
& \leq \sum_{q=1}^{H^{\sigma^{\prime \prime}}}\left(\frac{H^{\sigma^{\prime \prime}}-q+1}{H^{\sigma^{\prime \prime}}}\right)^{\left|\sigma_{j}^{\prime \prime}\right|-1} \cdot \frac{q-1}{H^{\sigma^{\prime \prime}}},
\end{aligned}
$$

implying $C_{j}\left(\sigma^{\prime \prime}\right)<C_{j}\left(\sigma_{-j}^{\prime \prime}, \sigma_{j}^{\prime \prime}-e\right)$, for all $e \in \sigma_{j}^{\prime \prime}$. Therefore, $\sigma^{\prime \prime}$ is D-stable and lies in $\Sigma^{2}$.

To show that $C_{N}\left(\sigma^{\prime \prime}\right)$, the group cost of $\sigma^{\prime \prime}$, satisfies $C_{N}\left(\sigma^{\prime \prime}\right)<C_{N}(\sigma)$, we note that $H^{\sigma^{\prime \prime}}=H^{\sigma}$, and thus $C_{k}\left(\sigma^{\prime \prime}\right)=C_{k}(\sigma)$, for all $k \in N \backslash\{i, j\}$. Therefore, we have to show that $C_{i}\left(\sigma^{\prime \prime}\right)+C_{j}\left(\sigma^{\prime \prime}\right)<$ $C_{i}(\sigma)+C_{j}(\sigma)$, or $C_{i}\left(\sigma^{\prime \prime}\right)-C_{i}(\sigma)<C_{j}(\sigma)-C_{j}\left(\sigma^{\prime \prime}\right)$. By Observation 4.1 .

$$
C_{i}\left(\sigma^{\prime}\right)<C_{i}(\sigma) \Rightarrow t<\sum_{q=1}^{H^{\sigma}}\left(\frac{H^{\sigma}-q+1}{H^{\sigma}}\right)^{\left|\sigma_{i}\right|} \cdot \frac{q-1}{H^{\sigma}+1},
$$

that, coupled with $\left[16\right.$, implies that $\left|\sigma_{j}\right|-1>\left|\sigma_{i}\right|$. Then,

$$
C_{i}\left(\sigma^{\prime \prime}\right)-C_{i}(\sigma)=t-\sum_{q=1}^{H^{\sigma}}\left(\frac{H^{\sigma}-q+1}{H^{\sigma}}\right)^{\left|\sigma_{i}\right|} \cdot \frac{q-1}{H^{\sigma}}<
$$




$$
t-\sum_{q=1}^{H^{\sigma}}\left(\frac{H^{\sigma}-q+1}{H^{\sigma}}\right)^{\left|\sigma_{j}\right|-1} \cdot \frac{q-1}{H^{\sigma}}=C_{j}(\sigma)-C_{j}\left(\sigma^{\prime \prime}\right)
$$

as required. Therefore, $\sigma^{\prime \prime}$ lies in $\Sigma^{2}$ and satisfies $C_{N}\left(\sigma^{\prime \prime}\right)<C_{N}(\sigma)$, in contradiction to $\sigma \in \Sigma^{3}$.

Hence, if $\sigma \in \Sigma^{3}$ then 15 holds for all $j \in N$ with $\left|\sigma_{j}\right|>1$ such that $\sigma_{j} \cap M(\sigma) \neq \varnothing$. Now let us see that there exists $\sigma \in \Sigma^{3}$ such that 15 holds for all the players. For that, choose a player $i \in \arg \max _{j \in N}\left|\sigma_{j}\right|$. If there exists $a \in \sigma_{i} \cap M(\sigma)$ then $i$ satisfies (15), implying by the choice of player $i$, that the above obviously yields the correctness of 15 for any player $k \in N$. Otherwise, if no resource in $\sigma_{i}$ lies in $M(\sigma)$, then let $a \in \sigma_{i}$ and $a^{\prime} \in M(\sigma)$. Since $a \in \sigma_{i}, a^{\prime} \notin \sigma_{i}$, and $h_{a}(\sigma)=h_{a^{\prime}}(\sigma)$, then there exists player $j$ such that $a^{\prime} \in \sigma_{j}$ and $a \notin \sigma_{j}$. One can easily check that the strategy profile $\sigma^{\prime}=\left(\sigma_{-\{i, j\}}, \sigma_{i}-a+a^{\prime}, \sigma_{j}-a^{\prime}+a\right)$ lies in $\Sigma^{3}$. Thus, $\sigma^{\prime}$ satisfies 15 for player $i$, and therefore, for any player $k \in N$.

Proof of Lemma 4.5. Using (1) with respect to $\sigma$, for any player $k$ with $\sigma_{k}^{\prime}=\sigma_{k}$ and for any $\sigma^{\prime}$-light resource $a \in \sigma_{k}^{\prime}$, we get

$$
\begin{aligned}
t & \leq \sum_{q=1}^{\min _{e \in \sigma_{k}-a} h_{e}(\sigma)}\left(\prod_{e \in \sigma_{k}-a} \frac{h_{e}(\sigma)-q+1}{h_{e}(\sigma)}\right) \frac{q-1}{h_{a}(\sigma)+1} \\
& \leq \sum_{q=1}^{\min _{e \in \sigma_{k}^{\prime}-a} h_{e}\left(\sigma^{\prime}\right)}\left(\prod_{e \in \sigma_{k}^{\prime}-a} \frac{h_{e}\left(\sigma^{\prime}\right)-q+1}{h_{e}\left(\sigma^{\prime}\right)}\right) \frac{q-1}{h_{a}\left(\sigma^{\prime}\right)+1},
\end{aligned}
$$

as required. Now let us consider the rest of the players. Assume $\sigma^{\prime}$ is obtained by the one-step addition of $a^{*}$ by player $i$. In this case, $i$ is the only player with $\sigma_{i}^{\prime} \neq \sigma_{i}$. The required property for player $i$ follows directly from $C_{i}\left(\sigma^{\prime}\right)<C_{i}(\sigma)$ :

$$
\sum_{q=1}^{\min _{e \in \sigma_{i}^{\prime}} h_{e}\left(\sigma^{\prime}\right)} \prod_{e \in \sigma_{i}^{\prime}} \frac{h_{e}\left(\sigma^{\prime}\right)-q+1}{h_{e}\left(\sigma^{\prime}\right)}+\left|\sigma_{i}^{\prime}\right| t<\sum_{q=1}^{\min _{e \in \sigma_{i}} h_{e}(\sigma)} \prod_{e \in \sigma_{i}} \frac{h_{e}(\sigma)-q+1}{h_{e}(\sigma)}+\left|\sigma_{i}\right| t .
$$

Since $h_{a^{*}}\left(\sigma^{\prime}\right)=h_{a^{*}}(\sigma)+1 \geq h_{e}(\sigma)=h_{e}\left(\sigma^{\prime}\right)$ for all $e \in M-a^{*}$ then $\min _{e \in \sigma_{i}^{\prime}} h_{e}\left(\sigma^{\prime}\right)=\min _{e \in \sigma_{i}+a^{*}} h_{e}\left(\sigma^{\prime}\right)=$ $\min _{e \in \sigma_{i}} h_{e}(\sigma)$, and by Observation 4.1 the above yields

$$
\begin{aligned}
t & <\sum_{q=1}^{\min _{e \in \sigma_{i}} h_{e}(\sigma)} \prod_{e \in \sigma_{i}}\left(\frac{h_{e}(\sigma)-q+1}{h_{e}(\sigma)}\right) \frac{q-1}{h_{a^{*}}(\sigma)+1} \\
& =\sum_{q=1}^{\min _{e \in \sigma_{i}^{\prime}-a^{*}} h_{e}\left(\sigma^{\prime}\right)}\left(\prod_{e \in \sigma_{i}^{\prime}-a^{*}} \frac{h_{e}\left(\sigma^{\prime}\right)-q+1}{h_{e}\left(\sigma^{\prime}\right)}\right) \frac{q-1}{h_{a^{*}}(\sigma)+1},
\end{aligned}
$$

implying

$$
t<\sum_{q=1}^{\min _{e \in \sigma_{i}^{\prime}-a} h_{e}\left(\sigma^{\prime}\right)}\left(\prod_{e \in \sigma_{i}^{\prime}-a} \frac{h_{e}\left(\sigma^{\prime}\right)-q+1}{h_{e}\left(\sigma^{\prime}\right)}\right) \frac{q-1}{h_{a}\left(\sigma^{\prime}\right)+1}
$$

for any $\sigma^{\prime}$-light resource $a$ (recall that $\min _{e \in M} h_{e}\left(\sigma^{\prime}\right)=\min _{e \in M} h_{e}(\sigma)$ ).

In the case of a two-step addition, let $\sigma^{\prime}=\left(\sigma_{-\{i, j\}}, \sigma_{i}+b^{*}, \sigma_{j}-b^{*}+a^{*}\right)$, where $b^{*}$ is a $\sigma$-heavy resource and $a^{*}$ is $\sigma$-light. For player $i, C_{i}\left(\sigma_{-i}, \sigma_{i}+b^{*}\right)<C_{i}(\sigma)$ coupled with $h_{b^{*}}(\sigma)+1>h_{e}(\sigma)$ and $h_{e}\left(\sigma^{\prime}\right) \geq h_{e}(\sigma)$ for all $e \in M-b^{*}$, yields

$$
\begin{aligned}
t & <\sum_{q=1}^{\min _{e \in \sigma_{i}} h_{e}(\sigma)} \prod_{e \in \sigma_{i}}\left(\frac{h_{e}(\sigma)-q+1}{h_{e}(\sigma)}\right) \frac{q-1}{h_{b^{*}}(\sigma)+1} \\
& \leq \sum_{q=1}^{\min _{e \in \sigma_{i}^{\prime}-b^{*}} h_{e}\left(\sigma^{\prime}\right)} \prod_{e \in \sigma_{i}^{\prime}-b^{*}}\left(\frac{h_{e}\left(\sigma^{\prime}\right)-q+1}{h_{e}\left(\sigma^{\prime}\right)}\right) \frac{q-1}{h_{b^{*}}(\sigma)+1} .
\end{aligned}
$$


Since $b^{*}$ is $\sigma$-heavy then $h_{b^{*}}(\sigma) \geq h_{e}\left(\sigma^{\prime}\right)$ for all $e \in M$ and, in particular, for all $\sigma^{\prime}$-light resources. Then, (17) implies

$$
t<\sum_{q=1}^{\min _{e \in \sigma_{i}^{\prime}-a} h_{e}\left(\sigma^{\prime}\right)} \prod_{e \in \sigma_{i}^{\prime}-a}\left(\frac{h_{e}\left(\sigma^{\prime}\right)-q+1}{h_{e}\left(\sigma^{\prime}\right)}\right) \frac{q-1}{h_{a}\left(\sigma^{\prime}\right)+1}
$$

for any $\sigma^{\prime}$-light resource $a$, as required.

For player $j$, the required follows directly from the D-stability of $\sigma$, coupled with $h_{b^{*}}(\sigma)=h_{a^{*}}\left(\sigma^{\prime}\right)=$ $h_{a}\left(\sigma^{\prime}\right)+1$ for any $\sigma^{\prime}$-light resource $a$ (the latter is true since $\min _{e \in M} h_{e}\left(\sigma^{\prime}\right)=\min _{e \in M} h_{e}(\sigma)$ and $b^{*}$ is $\sigma$-heavy):

$$
\begin{aligned}
t & \leq \sum_{q=1}^{\min _{e \in \sigma_{i}-b^{*}} h_{e}(\sigma)} \prod_{e \in \sigma_{i}-b^{*}}\left(\frac{h_{e}(\sigma)-q+1}{h_{e}(\sigma)}\right) \frac{q-1}{h_{b^{*}}(\sigma)} \\
& \leq \sum_{q=1}^{\min _{e \in \sigma_{i}^{\prime}-a} h_{e}\left(\sigma^{\prime}\right)} \prod_{e \in \sigma_{i}^{\prime}-a}\left(\frac{h_{e}\left(\sigma^{\prime}\right)-q+1}{h_{e}\left(\sigma^{\prime}\right)}\right) \frac{q-1}{h_{a}\left(\sigma^{\prime}\right)+1},
\end{aligned}
$$

as required.

Proof of TheOrem 5.1. First we prove that the RONE-algorithm finds a pure-strategy Nash equilibrium in a given $\mathrm{ROCG}$, and then proceed to the proof of its complexity.

Validity: In the simplest case where

$$
t \leq \sum_{q=1}^{n}\left(\frac{n-q+1}{n}\right)^{m-1} \frac{q-1}{n}
$$

the RONE-algorithm terminates after Step [0] with the outcome $\sigma=(M, \ldots, M)$ which is A- and S-stable. By Observation 4.1, (18) implies the D-stability of $\sigma$. Hence, by Lemma 4.1, $\sigma$ is a Nash equilibrium. Otherwise, if (18) does not hold, the algorithm proceeds to Step [1].

Consider first the case in which $t>\sum_{q=1}^{k}\left(\frac{k-q+1}{k}\right)^{\left\lceil\frac{k m}{n}\right\rceil-1} \frac{q-1}{k}$ for all $\left\lfloor\frac{n}{m}\right\rfloor<k \leq n-1$. In this case, the RONE-algorithm terminates after Step [2] with the output $\sigma=\left(e_{i \bmod m}\right)_{i \in N}$. Below we show that the above strategy profile is a Nash equilibrium. By Lemma 4.1. it suffices to prove the A-, D- and S-stability of $\sigma$. Since $\left|\sigma_{i}\right|=1$ for all $i \in N$ then no D-moves from $\sigma$ are available. In addition, since $\sigma$ is nearly-even, by Lemma 4.2 , it is S-stable as well. It remains to prove the A-stability of $\sigma$. That is, for any player $i \in N$ and resource $e \notin \sigma_{i}$ we have to show that $C_{i}(\sigma) \leq C_{i}\left(\sigma_{-i}, \sigma_{i}+e\right)$. Since $\sigma$ is nearly-even then $h_{e_{i \bmod m}}(\sigma) \leq h_{e}(\sigma)+1$ for all $e \in M$. Hence, by Observation 4.1, we need to show that

$$
t \geq \sum_{q=1}^{h_{e_{i \bmod m}}(\sigma)} \frac{h_{e_{i \bmod m}}(\sigma)-q+1}{h_{e_{i \bmod m}}(\sigma)} \cdot \frac{q-1}{h_{e}(\sigma)+1} .
$$

In $\sigma$, every resource has a congestion of $\left\lfloor\frac{n}{m}\right\rfloor$ or $\left\lceil\frac{n}{m}\right\rceil$, and every player uses precisely one resource. Therefore, to prove (19), it suffices to show that

$$
t \geq \sum_{q=1}^{\left\lceil\frac{n}{m}\right\rceil} \frac{\left\lceil\frac{n}{m}\right\rceil-q+1}{\left\lceil\frac{n}{m}\right\rceil} \cdot \frac{q-1}{\left\lfloor\frac{n}{m}\right\rfloor+1} .
$$

By the algorithm, $t>\sum_{q=1}^{k}\left(\frac{k-q+1}{k}\right)^{\left\lceil\frac{k m}{n}\right\rceil-1} \frac{q-1}{k}$ for any $\left\lfloor\frac{n}{m}\right\rfloor<k \leq n$ and, in particular, for $k=\left\lfloor\frac{n}{m}\right\rfloor+1$. That is,

$$
t>\sum_{q=1}^{\left\lfloor\frac{n}{m}\right\rfloor+1}\left(\frac{\left\lfloor\frac{n}{m}\right\rfloor-q+2}{\left\lfloor\frac{n}{m}\right\rfloor+1}\right)^{\left\lceil\frac{\left(\left\lfloor\frac{n}{m}\right\rfloor+1\right) m}{n}\right\rceil-1} \frac{q-1}{\left\lfloor\frac{n}{m}\right\rfloor+1} .
$$

Thus, 20 follows from $\sqrt[21]{ }$ by applying $\left\lfloor\frac{n}{m}\right\rfloor+1 \geq\left\lceil\frac{n}{m}\right\rceil$ and $\left\lceil\frac{\left(\left\lfloor\frac{n}{m}\right\rfloor+1\right) m}{n}\right\rceil-1 \leq 1$. 
In the complement case, the RONE-algorithm picks $\left\lfloor\frac{n}{m}\right\rfloor<k^{*} \leq n-1$ satisfying

$$
\begin{aligned}
t & \leq \sum_{q=1}^{k^{*}}\left(\frac{k^{*}-q+1}{k^{*}}\right)^{\left\lceil\frac{k^{*} m}{n}\right\rceil-1} \frac{q-1}{k^{*}} \\
\text { and } t & >\sum_{q=1}^{p}\left(\frac{p-q+1}{p}\right)^{\left\lceil\frac{p m}{n}\right\rceil-1} \frac{q-1}{p}
\end{aligned}
$$

for any $k^{*}<p \leq n$. At Step [3], the algorithm constructs a $k^{*}$-even strategy profile $\sigma$, in which $n^{*}=n\left(\left\lfloor\frac{k^{*} m}{n}\right\rfloor+1\right)-k^{*} m$ players use $\left\lfloor\frac{k^{*} m}{n}\right\rfloor$ resources and $n-n^{*}=k^{*} m-n\left\lfloor\frac{k^{*} m}{n}\right\rfloor$ players use $\left\lfloor\frac{k^{*} m}{n}\right\rfloor+1$ resources. That is, $\sigma$ is the most fair strategy profile among the set of $k^{*}$-even profiles (notice that the value of $n^{*}$ is determined by the equation $\left.n^{*}\left\lfloor\frac{k^{*} m}{n}\right\rfloor+\left(n-n^{*}\right)\left(\left\lfloor\frac{k^{*} m}{n}\right\rfloor+1\right)=k^{*} m\right)$. $\sigma$ is even and therefore is S-stable, by Lemma 4.2. Below we prove the D-stability of $\sigma$. We notice that if $n$ divides $k^{*} m$ then $n^{*}=n$ and $\left\lfloor\frac{k^{*} m}{n}\right\rfloor=\left\lceil\frac{k^{*} m}{n}\right\rceil$. Otherwise, $n^{*}<n$ and $\left\lfloor\frac{k^{*} m}{n}\right\rfloor+1=\left\lceil\frac{k^{*} m}{n}\right\rceil$. By Observation 4.1 22 is essentially equivalent to the inequality $C_{i}(\sigma) \leq C_{i}\left(\sigma_{-i}, \sigma_{i}-e\right)$ for any $i \in N$ with $\left|\sigma_{i}\right|=\left\lceil\frac{k^{*} m}{n}\right\rceil$ and $e \in \sigma_{i}$. In addition, since $\left\lfloor\frac{n}{m}\right\rfloor \leq\left\lceil\frac{n}{m}\right\rceil$, from 222 we get $t \leq \sum_{q=1}^{k^{*}}\left(\frac{k^{*}-q+1}{k^{*}}\right)^{\left\lfloor\frac{k^{*} m}{n}\right\rfloor-1} \frac{q-1}{k^{*}}$ which yields $C_{i}(\sigma) \leq C_{i}\left(\sigma_{-i}, \sigma_{i}-e\right)$ for any $i \in N$ with $\left|\sigma_{i}\right|=\left\lfloor\frac{k^{*} m}{n}\right\rfloor$ and $e \in \sigma_{i}$. Therefore, the strategy profile $\sigma$ is D-stable. Using similar arguments as before, one can see that if

$$
t \geq \sum_{q=1}^{k^{*}}\left(\frac{k^{*}-q+1}{k^{*}}\right)^{\left\lfloor\frac{k^{*} m}{n}\right\rfloor} \frac{q-1}{k^{*}+1}
$$

holds then, by Observation 4.1 (since all the resources are evenly congested), $\sigma$ is A-stable. Now, if (24) holds then the algorithm terminates after Step [4] with the outcome $\sigma$ which is a Nash equilibrium strategy profile, as follows from Lemma 4.1. Otherwise, if (24) does not hold, then the algorithm proceeds to Step [5]. In this case we show that $\sigma$ is post-addition D-stable and therefore can be turned into an equilibrium by applying on it sequentially at most $m-1$ one-/two-step addition operations (see Corollary 4.3.

Steps [5] - [9] describe the procedure of a one-/two-step addition operation. Since (24) does not hold then

$$
\begin{aligned}
t & <\sum_{q=1}^{k^{*}}\left(\frac{k^{*}-q+1}{k^{*}}\right)^{\left\lfloor\frac{k^{*} m}{n}\right\rfloor} \frac{q-1}{k^{*}+1} \\
& \leq \sum_{q=1}^{k^{*}}\left(\frac{k^{*}-q+1}{k^{*}}\right)^{\left\lfloor\frac{k^{*} m}{n}\right\rfloor-1} \frac{q-1}{k^{*}+1},
\end{aligned}
$$

implies that $\sigma$ is post-addition D-stable (see Definition 4.4). The algorithm halts at Step [6] if and only if the current strategy profile is A-stable. Otherwise, after Step [9] it results with a post-addition D-stable profile and proceeds to Step [5], and so on. Assume that the algorithm did not terminate after $m-1$ iterations of $[5]-[9]$. Then, at the $m$-th iteration, the algorithm produces an even strategy profile $\sigma$ with a common congestion of $k^{*}+1$. Therefore, there exists a player, say $i$, with $\left|\sigma_{i}\right| \geq\left\lceil\frac{\left(k^{*}+1\right) m}{n}\right\rceil$. By Lemma 4.5. $\sigma$ is D-stable. Then,

$$
\begin{aligned}
t & \leq \sum_{q=1}^{k^{*}+1}\left(\frac{k^{*}-q+2}{k^{*}+1}\right)^{\left|\sigma_{i}\right|-1} \frac{q-1}{k^{*}+1} \\
& \leq \sum_{q=1}^{k^{*}+1}\left(\frac{k^{*}-q+2}{k^{*}+1}\right)^{\left\lceil\frac{\left(k^{*}+1\right) m}{n}\right\rceil-1} \frac{q-1}{k^{*}+1}
\end{aligned}
$$

in contradiction to 23 . Therefore, Steps of [5] - [9] can be repeated at most $m-1$ times. Since the last iteration of [5] - [9] starts with a D-stable and, moreover, post-addition D-stable strategy profile, then the outcome of the algorithm is D-stable. The S-stability follows directly from Lemma 4.2 and a nearly even congestion of the resulting profile at each iteration. 
The above implies that the RONE-algorithm finds a pure strategy Nash equilibrium in a given ROCG with $m<n$.

Complexity: Step [0] takes $O(1)$ operations and is repeated only once. Step [1] takes $O(1)$ operations and can be repeated at most $n$ times. Steps [2], [3] and [4] takes $O(n), O(n m)$ and $O(1)$ operations, respectively, and can be repeated at most once each. Steps [5] - [9] take $O(n m)$ operations $-O(n m)$ at Step [5], $O(n)$ at Step [6], $O(m)$ at Step [7] and $O(n+m)$ at each of the Steps [8] and [9] - and can be repeated at most $m$ times. Therefore, the complexity of the RONE-algorithm is $O\left(\mathrm{~nm}^{2}\right)$.

7. Summary and Future Work. In this paper, we introduced and investigated the class of random order congestion games - ROCGs - which extends the models of congestion games to allow for a random ordering of task execution. In an ROCG, each player aims to minimize his own cost which is determined by the sum of two terms: the execution cost of his task which is assumed to be proportional to its completion time, and the sum of the fixed costs over the resources he uses. The completion time of the player's task is determined by the minimum among its completion times by all of his chosen resources.

We studied the existence of a pure strategy Nash equilibrium and a potential function in ROCGs. We showed that only ROCGs with 2 players and 2 resources are potential games, and any other ROCG is not a potential game. Nevertheless, we proved that any ROCG possesses a pure strategy Nash equilibrium. We presented a polynomial time algorithm for constructing a pure strategy Nash equilibrium in a given ROCG.

As indicated above, we proved the nonexistence of potential function for ROCGs. However, this does not preclude the existence of ordinal potential function, that if exists, insures that sequences of myopic moves by single players lead to equilibrium. We leave this interesting difficult issue for future work.

The model of ROCGs can be extended in various ways. One can consider other probability distributions over the set of permutations (orders) of the tasks assigned to a particular resource. In addition, it will be a challenge to consider different processing times rather than these of single units, different subsets of resources available to each of the players, players with multiple tasks etc. We believe such extensions will be significantly more difficult to analyze. It is also of interest to study the stability under deviations of coalitions and the social (in)efficiency of equilibria in ROCGs.

The method for computing a pure strategy Nash equilibrium proposed in this paper for ROCGs, uses the stability under single moves we presented in our previous paper on Congestion Games with Load-Dependent Failures [14. In future research we plan to consider more general settings with selfish players and shared resources, aiming at finding a characterization of classes of games for which the above technique is valid.

\section{References}

[1] E. Angel, E. Bampis, and F. Pascual. Truthful algorithms for scheduling selfish tasks on parallel machines. In WINE-05, pages 698-707, December 2005.

[2] V. Auletta, R. Prisco, P. Penna, and G. Persiano. Determinisitc truthful approximation mechanisms for scheduling related machines. In STACS-04, pages 608-619, 2004.

[3] T. Carroll and D. Grosu. Selfish multi-user task scheduling. In ISPDC-06, pages 99-106. IEEE Computer Society, 2006.

[4] G. Christodoulou and E. Koutsoupias. The price of anarchy of finite congestion games. In Proceedings of the 37th Annual ACM Symposium on Theory and Computing (STOC-05), pages 67-73, 2005.

[5] A. Fabrikant, C. Papadimitriou, and K. Talwar. The complexity of pure Nash equilibria. In STOC-04, pages 604-612, 2004.

[6] D. Grosu and T. Carroll. A strategyproof mechanism for scheduling divisible loads in distributed systems. In ISPDC-05, pages 83-90. IEEE Computer Society, July 2005.

[7] E. Koutsoupias. Selfish task allocation. Bulletin of EATCS, 81:79-88, October 2003.

[8] I. Milchtaich. Congestion games with player-specific payoff functions. Games and Economic Behavior, 13:111-124, 1996.

[9] D. Monderer. Solution-based congestion games. Advances in Mathematical Economics, 8:397-407, 2006. 
[10] D. Monderer and L. Shapley. Potential games. Games and Economic Behavior, 14:124-143, 1996.

[11] D. Monderer and M. Tennenholtz. Distributed games. Games and Economic Behavior, 28:181-188, 1999.

[12] N. Nisan and A. Ronen. Algorithmic mechanism design. Games and Economic Behavior, 35(1/2):166-196, April 2001.

[13] M. Penn, M. Polukarov, and M. Tennenholtz. Congestion games with failures. In Proceedings of the 6th ACM Conference on Electronic Commerce (EC-05), pages 259-268, 2005.

[14] M. Penn, M. Polukarov, and M. Tennenholtz. Congestion games with load-dependent failures: identical resources. In Proceedings of the 8th ACM Conference on Electronic Commerce (EC-07), pages 210-217, 2007.

[15] M. Penn, M. Polukarov, and M. Tennenholtz. Asynchronous congestion games. In Proc. of 7th Int. Conf. on Autonomous Agents and Multiagent Systems (AAMAS-08), pages 1605-1608, May 2008.

[16] R. Rosenthal. A class of games possessing pure-strategy Nash equilibria. International Journal of Game Theory, 2:65-67, 1973. 